\title{
Characters and chromatic symmetric functions
}

\author{
Mark Skandera \\ Department of Mathematics \\ Lehigh University \\ Bethlehem, PA, U.S.A. \\ mas906@lehigh.edu
}

Submitted: Jul 17, 2020; Accepted: Feb 20, 2021; Published: May 7, 2021

(C) The author. Released under the CC BY-ND license (International 4.0).

\begin{abstract}
Given a poset $P$, let inc $(P)$ be its incomparability graph, and $X_{\operatorname{inc}(P)}$ the corresponding chromatic symmetric function defined by Stanley in Adv. Math., 111 (1995) pp. 166-194. Let $\omega$ be the standard involution on symmetric functions. We express coefficients of $X_{\operatorname{inc}(P)}$ and $\omega X_{\operatorname{inc}(P)}$ as character evaluations to obtain simple combinatorial interpretations for coefficients of the power sum and monomial expansions of $\omega X_{\operatorname{inc}(P)}$ which hold for all posets $P$. Consequences include new combinatorial interpretations of the permanent, induced trivial character immanants, and power sum immanants of totally nonnegative matrices, and of the sum of elementary coefficients in the Shareshian-Wachs chromatic quasisymmetric function $X_{\text {inc }(P), q}$ when $P$ is an appropriately labeled unit interval order.
\end{abstract}

Mathematics Subject Classifications: 05E05, 05C15, 15C15, 15A15, $20 \mathrm{C} 08$

\section{Introduction}

The Frobenius isomorphism from the space $\mathcal{T}_{n}$ of symmetric group traces to the space $\Lambda_{n}$ of homogeneous degree- $n$ symmetric functions,

$$
\text { Frob } \begin{aligned}
: \mathcal{T}_{n} & \rightarrow \Lambda_{n} \\
\theta & \mapsto \frac{1}{n !} \sum_{w \in \mathfrak{S}_{n}} \theta(w) p_{\operatorname{ctype}(w),}
\end{aligned}
$$

where $\operatorname{ctype}(w)$ is the cycle type of $w$, allows one to translate statements about the representation theory of the symmetric group $\mathfrak{S}_{n}$ to the language of symmetric functions. Conversely, one may use the inverse of the Frobenius isomorphism to study symmetric functions, such as Stanley's chromatic symmetric functions $X_{G}$ [30], in terms of $\mathfrak{S}_{n^{-}}$ class functions. In particular, for $G$ the incomparability graph $\operatorname{inc}(P)$ of a poset $P$, we 
will expand $X_{G}$ in the standard symmetric function bases, and we will use the inverse Frobenius isomorphism to interpret the resulting coefficients. Our main tool is reminiscent of the Cauchy and dual Cauchy identities $[24, \S I .4]$ for symmetric functions in two sets of variables,

$$
\begin{aligned}
\prod_{i, j \geqslant 1} \frac{1}{1-y_{i} x_{j}}=\sum_{\lambda} e_{\lambda}(y) f_{\lambda}(x) & =\sum_{\lambda} h_{\lambda}(y) m_{\lambda}(x)=\sum_{\lambda} \frac{p_{\lambda}(y)}{z_{\lambda}} p_{\lambda}(x) \\
= & \sum_{\lambda} s_{\lambda}(y) s_{\lambda}(x)=\sum_{\lambda} m_{\lambda}(y) h_{\lambda}(x)=\sum_{\lambda} f_{\lambda}(y) e_{\lambda}(x), \\
\prod_{i, j \geqslant 1}\left(1+y_{i} x_{j}\right)=\sum_{\lambda} e_{\lambda}(y) m_{\lambda}(x) & =\sum_{\lambda} h_{\lambda}(y) f_{\lambda}(x)=\sum_{\lambda} \frac{(-1)^{n-\ell(\lambda)} p_{\lambda}(y)}{z_{\lambda}} p_{\lambda}(x) \\
= & \sum_{\lambda} s_{\lambda^{\top}}(y) s_{\lambda}(x)=\sum_{\lambda} m_{\lambda}(y) e_{\lambda}(x)=\sum_{\lambda} f_{\lambda}(y) h_{\lambda}(x),
\end{aligned}
$$

with the inverse Frobenius isomorphism applied only to the symmetric functions in $y$.

In Section 2 we present standard bases of the trace space of the Hecke algebra $H_{n}(q)$, the trace space of the symmetric group algebra $\mathbb{Z}\left[\mathfrak{S}_{n}\right]$, and the space $\Lambda_{n}$ of homogeneous degree- $n$ symmetric functions. We show that the expansion of any homogeneous degree- $n$ symmetric function in any standard basis of $\Lambda_{n}$ yields coefficients which are trace evaluations. In Section 3 we apply this result to the standard expansions of chromatic symmetric functions of the form $X_{\operatorname{inc}(P)}$ and the related symmetric functions $\omega X_{\operatorname{inc}(P)}$. We obtain combinatorial interpretations for resulting coefficients of $X_{\operatorname{inc}(P)}$ and $\omega X_{\operatorname{inc}(P)}$ for all posets $P$, thus extending previous results which hold only for special classes of posets. In particular, we interpret the monomial and power sum coefficients of $\omega X_{\operatorname{inc}(P)}$ in Theorems 10 and 13, respectively. We also obtain a new proof in Proposition 8 of Kaliszewski's interpretation of hook-Schur coefficients. In each case, the trace evaluations allow for very simple proofs of our results. In Section 4 we apply our interpretations of trace evaluations to functions of totally nonnegative matrices, obtaining new interpretations of these. In particular, we interpret "hook" irreducible character immanants in Theorem 27, induced trivial character immanants in Theorem 31, and power sum immanants in Theorem 32. These results include two new interpretations (55) - (56) of the permanent of a totally nonnegative matrix and play an important role in the evaluation of hyperoctahedral group characters at elements of the type- $B C$ Kazhdan-Lusztig basis [29]. These results also lead to a new expression in Section 5 for the sum of elementary coefficients of the Shareshian-Wachs chromatic quasisymmetric function $X_{\operatorname{inc}(P), q}$ when $P$ is a unit interval order.

\section{Symmetric functions and traces}

Let $\Lambda$ be the ring of symmetric functions in $x=\left(x_{1}, x_{2}, \ldots\right)$ having integer coefficients, and let $\Lambda_{n}$ be the $\mathbb{Z}$-submodule of homogeneous functions of degree $n$. This submodule 
has rank equal to the number of integer partitions of $n$, the weakly decreasing positive integer sequences $\lambda=\left(\lambda_{1}, \ldots, \lambda_{\ell}\right)$ satisfying $\lambda_{1}+\cdots+\lambda_{\ell}=n$. The $\ell=\ell(\lambda)$ components of $\lambda$ are called its parts, and we let $|\lambda|=n$ and $\lambda \vdash n$ denote that $\lambda$ is a partition of $n$. Given $\lambda \vdash n$, we define the transpose partition $\lambda^{\top}=\left(\lambda_{1}^{\top}, \ldots, \lambda_{\lambda_{1}}^{\top}\right)$ by

$$
\lambda_{i}^{\top}=\#\left\{j \mid \lambda_{j} \geqslant i\right\}
$$

Sometimes it is convenient to name a partition with exponential notation, omitting parentheses and commas, so that $4^{2} 1^{6}:=(4,4,1,1,1,1,1,1)$. We define a composition of $n$ to be any rearrangement of a partition of $n$ and write $\alpha \vDash n$ to denote that $\alpha$ is a composition of $n$. Six standard bases of $\Lambda_{n}$ consist of the monomial $\left\{m_{\lambda} \mid \lambda \vdash n\right\}$, elementary $\left\{e_{\lambda} \mid \lambda \vdash n\right\}$, (complete) homogenous $\left\{h_{\lambda} \mid \lambda \vdash n\right\}$, power sum $\left\{p_{\lambda} \mid \lambda \vdash n\right\}$, Schur $\left\{s_{\lambda} \mid \lambda \vdash n\right\}$, and forgotten $\left\{f_{\lambda} \mid \lambda \vdash n\right\}$ symmetric functions. (See, e.g., [32, Ch.7] for definitions.) An involutive automorphism $\omega: \Lambda \rightarrow \Lambda$ defined by $\omega\left(e_{k}\right)=h_{k}$ for all $k$ acts on these bases of $\Lambda_{n}$ by

$$
\omega\left(s_{\lambda}\right)=s_{\lambda^{\top}}, \quad \omega\left(m_{\lambda}\right)=f_{\lambda}, \quad \omega\left(e_{\lambda}\right)=h_{\lambda}, \quad \omega\left(p_{\lambda}\right)=(-1)^{n-\ell(\lambda)} p_{\lambda} .
$$

Let $H_{n}(q)$ be the (type $A$ ) Hecke algebra, generated over $\mathbb{Z}\left[q^{\frac{1}{2}}, q^{-\frac{1}{2}}\right]$ by $T_{s_{1}}, \ldots, T_{s_{n-1}}$ subject to relations

$$
\begin{aligned}
T_{s_{i}}^{2} & =(q-1) T_{s_{i}}+q & & \text { for } i=1, \ldots, n-1, \\
T_{s_{i}} T_{s_{j}} & =T_{s_{j}} T_{s_{i}} & & \text { for }|i-j| \geqslant 2, \\
T_{s_{i}} T_{s_{j}} T_{s_{i}} & =T_{s_{j}} T_{s_{i}} T_{s_{j}} & & \text { for }|i-j|=1 .
\end{aligned}
$$

For each $w \in \mathfrak{S}_{n}$ and $w=s_{i_{1}} \cdots s_{i_{\ell}}$ a reduced expression, define the natural basis element $T_{w}=T_{s_{i_{1}}} \cdots T_{s_{i_{\ell}}}$ (which does not depend upon the choice of a reduced expression). (See, e.g., [3].) The (modified) Kazhdan-Lusztig basis of $H_{n}(q)$ as a $\mathbb{Z}\left[q^{\frac{1}{2}}, q^{-\frac{1}{2}}\right]$-module consists of elements $\left\{\widetilde{C}_{w}(q) \mid w \in \mathfrak{S}_{n}\right\}$ related to the natural basis by

$$
\widetilde{C}_{w}(q)=\sum_{v \leqslant w} P_{v, w}(q) T_{v}
$$

where $\leqslant$ is the Bruhat order on $\mathfrak{S}_{n}$, and where $\left\{P_{v, w}(q) \mid v, w \in \mathfrak{S}_{n}\right\}$ are the recursively defined Kazhdan-Lusztig polynomials. (Our basis element $\widetilde{C}_{w}(q)$ is $q^{\frac{\ell(w)}{2}}$ times the basis element $C_{w}^{\prime}$ in [20].) When $w$ avoids the patterns 3412 and 4231 (the one-line notation $w_{1} \cdots w_{n}$ contains no subword $w_{i_{1}} w_{i_{2}} w_{i_{3}} w_{i_{4}}$ whose letters have values appearing in the same relative order as 4231 or 3412 ), each polynomial $P_{v, w}(q)$ is identically 1.

Let $\mathcal{T}_{n, q}$ be the $\mathbb{Z}\left[q^{\frac{1}{2}}, q^{\frac{1}{2}}\right]$-module of $H_{n}(q)$-traces, linear functionals $\theta_{q}: H_{n}(q) \rightarrow$ $\mathbb{Z}\left[q^{\frac{1}{2}}, q^{-\frac{1}{2}}\right]$ satisfying $\theta_{q}(g h)=\theta_{q}(h g)$ for all $g, h \in H_{n}(q)$. For any trace $\theta_{q}: T_{w} \mapsto a(q)$ in $\mathcal{T}_{n, q}$, the $q^{\frac{1}{2}}=1$ specialization $\theta: w \mapsto a(1)$ belongs to the space $\mathcal{T}_{n}:=\mathcal{T}_{n, 1}$ of $\mathbb{Z}\left[\mathfrak{S}_{n}\right]$-traces from $\mathbb{Z}\left[\mathfrak{S}_{n}\right] \rightarrow \mathbb{Z}\left(\mathfrak{S}_{n}\right.$-class functions). Like the $\mathbb{Z}$-module $\Lambda_{n}$, the trace spaces $\mathcal{T}_{n, q}$ and $\mathcal{T}_{n}$ have dimension equal to the number of integer partitions of $n$. The Frobenius $\mathbb{Z}$-module isomorphism (1) and its $q$-extension, $\operatorname{Frob}_{q}: \mathcal{T}_{n, q} \rightarrow \Lambda_{n}, \theta_{q} \mapsto \operatorname{Frob}(\theta)$, define bijections 
between standard bases of $\Lambda, \mathcal{T}_{n}$, and $\mathcal{T}_{n, q}$. Schur functions correspond to irreducible characters,

$$
s_{\lambda} \leftrightarrow \chi^{\lambda} \leftrightarrow \chi_{q}^{\lambda},
$$

while elementary and homogeneous symmetric functions correspond to induced sign and trivial characters,

$$
\begin{gathered}
e_{\lambda} \leftrightarrow \epsilon^{\lambda}=\operatorname{sgn} \uparrow_{\mathfrak{S}_{\lambda}}^{\mathfrak{S}_{n}} \leftrightarrow \epsilon_{q}^{\lambda}=\operatorname{sgn}_{q} \uparrow_{H_{\lambda}(q)}^{H_{n}(q)}, \\
h_{\lambda} \leftrightarrow \eta^{\lambda}=\operatorname{triv} \uparrow_{\mathfrak{S}_{\lambda}}^{\mathfrak{S}_{\lambda}} \leftrightarrow \eta_{q}^{\lambda}=\operatorname{triv}_{q} \uparrow_{H_{\lambda}(q)}^{H_{n}(q)},
\end{gathered}
$$

where $\mathfrak{S}_{\lambda}$ is the Young subgroup of $\mathfrak{S}_{n}$ indexed by $\lambda$ and $H_{\lambda}(q)$ is the corresponding parabolic subalgebra of $H_{n}(q)$. The power sum, monomial, and forgotten bases of $\Lambda_{n}$ correspond to bases of $\mathcal{T}_{n}\left(\mathcal{T}_{n, q}\right)$ which are not characters. We call these the power sum $\left\{\psi^{\lambda} \mid \lambda \vdash n\right\}\left(\left\{\psi_{q}^{\lambda} \mid \lambda \vdash n\right\}\right)$, monomial $\left\{\phi^{\lambda} \mid \lambda \vdash n\right\}\left(\left\{\phi_{q}^{\lambda} \mid \lambda \vdash n\right\}\right)$, and forgotten $\left\{\gamma^{\lambda} \mid \lambda \vdash\right.$ $n\}\left(\left\{\gamma_{q}^{\lambda} \mid \lambda \vdash n\right\}\right)$ traces, respectively. Specifically, these are the bases related to the irreducible character bases by the same matrices of character evaluations and inverse Koskta numbers that relate power sum, monomial, and forgotten symmetric functions to Schur functions,

$$
\begin{aligned}
& p_{\lambda}=\sum_{\mu} \chi^{\mu}(\lambda) s_{\mu}, \quad \psi^{\lambda}=\sum_{\mu} \chi^{\mu}(\lambda) \chi^{\mu}, \quad \psi_{q}^{\lambda}=\sum_{\mu} \chi^{\mu}(\lambda) \chi_{q}^{\mu}, \\
& m_{\lambda}=\sum_{\mu} K_{\lambda, \mu}^{-1} s_{\mu}, \quad \phi^{\lambda}=\sum_{\mu} K_{\lambda, \mu}^{-1} \chi^{\mu}, \quad \phi_{q}^{\lambda}=\sum_{\mu} K_{\lambda, \mu}^{-1} \chi_{q}^{\mu}, \\
& f_{\lambda}=\sum_{\mu} K_{\lambda, \mu^{\top}}^{-1} s_{\mu}, \quad \gamma^{\lambda}=\sum_{\mu} K_{\lambda, \mu^{\top}}^{-1} \chi^{\mu}, \quad \gamma_{q}^{\lambda}=\sum_{\mu} K_{\lambda, \mu^{\top}}^{-1} \chi_{q}^{\mu},
\end{aligned}
$$

where $\chi^{\mu}(\lambda):=\chi^{\mu}(w)$ for any $w \in \mathfrak{S}_{n}$ having $\operatorname{ctype}(w)=\lambda$. Just as the power sum symmetric functions form a $\mathbb{Q}$-basis of $\Lambda_{n}$, the power sum traces form $\mathbb{Q}$-bases of $\mathcal{T}_{n}$ and $\mathcal{T}_{n, q}$. The power sum traces of $\mathcal{T}_{n}$ also have the natural definition

$$
\psi^{\lambda}(w):= \begin{cases}z_{\lambda} & \text { if } \operatorname{ctype}(w)=\lambda \\ 0 & \text { otherwise }\end{cases}
$$

where $z_{\lambda}=\lambda_{1} \cdots \lambda_{\ell} \alpha_{1} ! \cdots \alpha_{n}$ ! and $\alpha_{i}$ is the number of parts of $\lambda$ equal to $i$.

It can be useful to record trace evaluations in a symmetric generating function. In particular, for $g \in \mathbb{Q}(q) \otimes H_{n}(q)$, we record induced sign character evaluations by defining

$$
Y_{q}(g):=\sum_{\lambda \vdash n} \epsilon_{q}^{\lambda}(g) m_{\lambda} \in \mathbb{Q}(q) \otimes \Lambda_{n} .
$$

This symmetric generating function in fact gives us all of the standard trace evaluations.

Proposition 1. The symmetric function $Y_{q}(g)$ is equal to

$$
\sum_{\lambda \vdash n} \eta_{q}^{\lambda}(g) f_{\lambda}=\sum_{\lambda \vdash n} \frac{(-1)^{n-\ell(\lambda)} \psi_{q}^{\lambda}(g)}{z_{\lambda}} p_{\lambda}=\sum_{\lambda \vdash n} \chi_{q}^{\lambda^{\top}}(g) s_{\lambda}=\sum_{\lambda \vdash n} \phi_{q}^{\lambda}(g) e_{\lambda}=\sum_{\lambda \vdash n} \gamma_{q}^{\lambda}(g) h_{\lambda} ;
$$


equivalently, $\omega Y_{q}(g)$ is equal to

$$
\sum_{\lambda \vdash n} \epsilon_{q}^{\lambda}(g) f_{\lambda}=\sum_{\lambda \vdash n} \eta_{q}^{\lambda}(g) m_{\lambda}=\sum_{\lambda \vdash n} \frac{\psi_{q}^{\lambda}(g)}{z_{\lambda}} p_{\lambda}=\sum_{\lambda \vdash n} \chi_{q}^{\lambda}(g) s_{\lambda}=\sum_{\lambda \vdash n} \phi_{q}^{\lambda}(g) h_{\lambda}=\sum_{\lambda \vdash n} \gamma_{q}^{\lambda}(g) e_{\lambda} .
$$

While this follows from (2) - (3), we provide a short proof.

Proof. Consider the second and fourth sums in (8), in which the symmetric functions and traces satisfy

$$
s_{\lambda}=\sum_{\mu \vdash n} K_{\lambda, \mu} m_{\mu}, \quad \eta_{q}^{\mu}=\sum_{\lambda \vdash n} K_{\lambda, \mu} \chi_{q}^{\lambda} .
$$

Using (9) to expand the fourth sum in the monomial symmetric function basis, we have

$$
\sum_{\lambda \vdash n} \chi_{q}^{\lambda}(g) \sum_{\mu \vdash n} K_{\lambda, \mu} m_{\mu}=\sum_{\mu \vdash n} \sum_{\lambda \vdash n} K_{\lambda, \mu} \chi_{q}^{\lambda}(g) m_{\mu}=\sum_{\mu \vdash n} \eta_{q}^{\mu}(g) m_{\mu},
$$

i.e., it is equal to the second sum. Similarly, for each of the remaining sums $\sum_{\lambda} \theta_{q}^{\lambda}(g) t_{\lambda}$ in (8), there is a matrix $\left(M_{\lambda, \mu}\right)_{\lambda, \mu \vdash n}$ and equations

$$
s_{\lambda}=\sum_{\mu \vdash n} M_{\lambda, \mu} t_{\mu}, \quad \theta_{q}^{\mu}=\sum_{\lambda \vdash n} M_{\lambda, \mu} \chi_{q}^{\lambda},
$$

relating it to the fourth sum. In particular, $M_{\lambda, \mu}=K_{\lambda^{\top}, \mu}, \chi^{\lambda}(\mu), K_{\mu, \lambda}^{-1}, K_{\mu, \lambda^{\top}}^{-1}$, respectively. (See $[2, \S 2]$.

Since each symmetric function is a quasisymmetric function, researchers sometimes express elements of $\Lambda_{n}$ in terms of bases of the $\mathbb{Z}$-module QSym $_{n}$ of degree- $n$ quasisymmetric functions. (See $[32, \S 7.19]$ for definitions). The coefficients arising in such expansions also can be viewed as trace evaluations. In particular, let $\left\{F_{n, S} \mid S \subseteq[n-1]\right\}$ be the fundamental quasisymmetric function basis of QSym $_{n}$. For any Young tableau $U$ of shape $\lambda=\left(\lambda_{1}, \ldots, \lambda_{r}\right)$, let $U_{1}, \ldots, U_{r}$ denote its rows, and let $\circ$ denote concatenation of rows. Define the inverse descent set of $U$ by

$$
\operatorname{IDES}(U)=\left\{i \in[n-1] \mid i+1 \text { appears before } i \text { in } U_{r} \circ \cdots \circ U_{1}\right\} .
$$

Now we have the following fundamental quasisymmetric expansion of $Y_{q}(g)$.

Corollary 2. For $\lambda=\left(\lambda_{1}, \ldots, \lambda_{r}\right) \vdash n$ and $S \subseteq[n-1]$, define $b(\lambda, S)$ to be the number of standard Young tableaux $U$ of shape $\lambda$ with $\operatorname{IDES}(U)=S$. Then we have

$$
Y_{q}(g)=\sum_{S \subseteq[n-1]} \sum_{\lambda \vdash n} b(\lambda, S) \chi_{q}^{\lambda^{\top}}(g) F_{n, S} ; \quad \omega Y_{q}(g)=\sum_{S \subseteq[n-1]} \sum_{\lambda \vdash n} b(\lambda, S) \chi_{q}^{\lambda}(g) F_{n, S} .
$$


Proof. By [13, Thm. 11], the coefficients $\left\{c_{\lambda} \mid \lambda \vdash n\right\}$ and $\left\{d_{S} \mid S \subseteq[n-1]\right\}$ appearing in the Schur and fundamental expansions

$$
\sum_{\lambda \vdash n} c_{\lambda} s_{\lambda}=\sum_{S \subseteq[n-1]} d_{S} F_{n, S}
$$

of a symmetric function satisfy

$$
d_{S}=\sum_{\lambda \vdash n} b(\lambda, S) c_{\lambda}
$$

The result now follows from Proposition 1.

To say that the functions $\left\{Y_{q}(g) \mid g \in H_{n}(q)\right\}$ arise often in the study of symmetric functions would be an understatement; essentially every element of $\mathbb{Z}[q] \otimes \Lambda_{n}$ has this form.

Proposition 3. Every symmetric function in $\mathbb{Z}[q] \otimes \Lambda_{n}$ has the form $Y_{q}(g)$ for some element $g \in \mathbb{Q}(q) \otimes H_{n}(q)$.

Proof. Fix a symmetric function in $\mathbb{Z}[q] \otimes \Lambda_{n}$, express it in the elementary basis as $\sum_{\lambda \vdash n} a_{\lambda} e_{\lambda}$, and define the $H_{n}(q)$ element

$$
g=\sum_{\mu \vdash n} \frac{a_{\mu}}{\left[\mu_{1}\right]_{q} ! \cdots\left[\mu_{\ell(\mu)}\right]_{q} !} \widetilde{C}_{w_{\mu}}(q)
$$

where

$$
[b]_{q}:=\left\{\begin{array}{ll}
1+q+\cdots+q^{b-1} & \text { if } b \geqslant 1, \\
0 & \text { if } b=0 ;
\end{array} \quad[b]_{q} !:= \begin{cases}{[1]_{q}[2]_{q} \cdots[b]_{q}} & \text { if } b \geqslant 1, \\
1 & \text { if } b=0\end{cases}\right.
$$

and $w_{\mu}$ is the maximal element of the Young subgroup $\mathfrak{S}_{\mu}$ of $\mathfrak{S}_{n}$. By [16, Prop. 4.1], we have

$$
\phi_{q}^{\lambda}\left(\widetilde{C}_{w_{\mu}}(q)\right)= \begin{cases}{\left[\mu_{1}\right]_{q} ! \cdots\left[\mu_{\ell(\mu)}\right]_{q} !} & \text { if } \lambda=\mu \\ 0 & \text { otherwise }\end{cases}
$$

Thus $Y_{q}(g)$ is equal to

$$
\sum_{\lambda \vdash n} \phi_{q}^{\lambda}\left(\sum_{\mu \vdash n} \frac{a_{\mu}}{\left[\mu_{1}\right]_{q} ! \cdots\left[\mu_{\ell(\mu)}\right]_{q} !} \widetilde{C}_{w_{\mu}}(q)\right) e_{\lambda}=\sum_{\lambda \vdash n} \sum_{\mu \vdash n} a_{\mu} \frac{\phi_{q}^{\lambda}\left(\widetilde{C}_{w_{\mu}}(q)\right)}{\left[\mu_{1}\right]_{q} ! \cdots\left[\mu_{\ell(\mu)}\right]_{q} !} e_{\lambda}=\sum_{\lambda \vdash n} a_{\lambda} e_{\lambda} .
$$

Of course, for $g \in \mathbb{Q}\left[\mathfrak{S}_{n}\right]$, the $q^{\frac{1}{2}}=1$ specialization $Y(g):=Y_{1}(g)$ of $(7)$ satisfies the $q^{\frac{1}{2}}=1$ specializations of Proposition 1, Corollary 2, and Proposition 3. 


\section{Chromatic symmetric functions}

Closely related to symmetric generating functions for $\mathbb{Z}\left[\mathfrak{S}_{n}\right]$-traces are symmetric generating functions for graph colorings. Define a proper coloring of a (simple undirected) graph $G=(V, E)$ to be an assignment $\kappa: V \rightarrow\{1,2, \ldots$,$\} of colors (positive integers)$ to $V$ such that adjacent vertices have different colors. For $G$ on $|V|=n$ vertices and any composition $\alpha=\left(\alpha_{1}, \ldots, \alpha_{\ell}\right) \vDash n$, say that a coloring $\kappa$ of $G$ has type $\alpha$ if $\alpha_{i}$ vertices have color $i$ for $i=1, \ldots, \ell$. Let $c(G, \alpha)$ be the number of proper colorings of $G$ of type $\alpha$. Stanley [30] defined the chromatic symmetric function of $G$ to be

$$
X_{G}:=\sum_{\kappa} x_{\kappa(1)} \cdots x_{\kappa(n)}=\sum_{\lambda \vdash n} c(G, \lambda) m_{\lambda}
$$

where the first sum is over all proper colorings of $G$. By Proposition 3 we see that for each graph $G$ on $n$ vertices, there exists an element $g \in \mathbb{Q}\left[\mathfrak{S}_{n}\right]$ such that $X_{G}=Y(g)$. Such an element $g$ is not uniquely determined by $G$, and is not in general easily described in terms of the structure of $G$. On the other hand, the evaluations of traces at such elements are easily described in terms of $G$.

Observation 4. Let $G$ be a graph on $n$ vertices and let $g \in \mathbb{Q}\left[\mathfrak{S}_{n}\right]$ satisfy $Y(g)=X_{G}$. Then for each trace $\theta=\sum_{\lambda \vdash n} a_{\lambda} \epsilon^{\lambda} \in \mathcal{T}_{n}$, we have $\theta(g)=\sum_{\lambda \vdash n} a_{\lambda} c(G, \lambda)$.

Proof. By (7) and (11) we have $\epsilon^{\lambda}(g)=c(G, \lambda)$ for each $\lambda \vdash n$.

For every trace $\theta \in \mathcal{T}_{n}$, Proposition 3 and Observation 4 allow us to define

$$
\theta(G):=\theta(g)
$$

where $g$ is any element in $\mathbb{Q}\left[\mathfrak{S}_{n}\right]$ satisfying $Y(g)=X_{G}$. By Proposition 1 , we have that $X_{G}=\sum_{\lambda \vdash n} \epsilon^{\lambda}(G) m_{\lambda}$ is equal to

$$
\sum_{\lambda \vdash n} \eta^{\lambda}(G) f_{\lambda}=\sum_{\lambda \vdash n} \frac{(-1)^{n-\ell(\lambda)} \psi^{\lambda}(G)}{z_{\lambda}} p_{\lambda}=\sum_{\lambda \vdash n} \chi^{\lambda^{\top}}(G) s_{\lambda}=\sum_{\lambda \vdash n} \phi^{\lambda}(G) e_{\lambda}=\sum_{\lambda \vdash n} \gamma^{\lambda}(G) h_{\lambda} ;
$$

equivalently, $\omega X_{G}$ is equal to

$$
\sum_{\lambda \vdash n} \epsilon^{\lambda}(G) f_{\lambda}=\sum_{\lambda \vdash n} \eta^{\lambda}(G) m_{\lambda}=\sum_{\lambda \vdash n} \frac{\psi^{\lambda}(G)}{z_{\lambda}} p_{\lambda}=\sum_{\lambda \vdash n} \chi^{\lambda}(G) s_{\lambda}=\sum_{\lambda \vdash n} \phi^{\lambda}(G) h_{\lambda}=\sum_{\lambda \vdash n} \gamma^{\lambda}(G) e_{\lambda} .
$$

Some conditions on graphs $G$ and traces $\theta$ imply the numbers $\theta(G)$ to be positive, and sometimes the resulting positive numbers have nice combinatorial interpretations, particularly when $G$ is the incomparability graph of a poset. (See, e.g., [8], [27], [30].) Given a poset $P$, define its incomparability graph $\operatorname{inc}(P)$ to be the graph having a vertex for each element of $P$ and an edge $\{i, j\}$ for each incomparable pair of elements of $P$. For positive integers $a, b$, call a poset $(\mathbf{a}+\mathbf{b})$-free if it has no induced subposet isomorphic to a disjoint sum of an $a$-element 
chain and a $b$-element chain. For example, the following poset $P$ is $(\mathbf{3}+\mathbf{1})$-free and $(\mathbf{2}+\mathbf{2})$-free, and has incomparability graph $\operatorname{inc}(P)=G$.

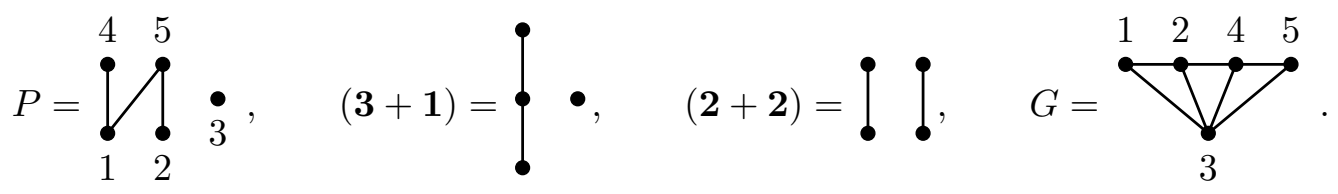

If we cannot interpret $\theta(\operatorname{inc}(P))$ for all posets $P$, sometimes we can do so when $P$ is $(\mathbf{3}+\mathbf{1})$ free. For an $n$-element poset $P$ which is both $(\mathbf{3}+\mathbf{1})$-free and $(\mathbf{2}+\mathbf{2})$-free, also called a unit interval order, a simple procedure produces an element $g \in \mathfrak{S}_{n}$ satisfying $Y(g)=X_{\text {inc }(P)}$. Explicitly, for each element $y \in P$, compute

$$
\beta(y):=\#\left\{x \in P \mid x \leqslant_{P} y\right\}-\#\left\{z \in P \mid z \geqslant_{P} y\right\}
$$

and label the poset elements $1, \ldots, n$ so that we have

$$
\beta(1) \leqslant \cdots \leqslant \beta(n) .
$$

Then define $w=w(P)=w_{1} \cdots w_{n}$ by

$$
w_{j}=\max \left(\left\{i \mid i \ngtr_{P} j\right\} \backslash\left\{w_{1}, \ldots, w_{j-1}\right\}\right) .
$$

For example, elements of the poset $P$ in (15) are already labeled to satsify (16),

$$
(\beta(1), \beta(2), \beta(3), \beta(4), \beta(5))=(-2,-1,0,1,2) .
$$

Thus we compute $w(P)=34521$. The labeling (16) of $P$ is natural in the sense that elements labeled $a_{1}, a_{2}$ satisfy

$$
a_{1}<_{P} a_{2} \quad \Longrightarrow \quad a_{1}<a_{2} \text { (as integers). }
$$

The map $P \mapsto w(P)$ is a bijection from $n$-element unit interval orders to the $\frac{1}{n+1}\left(\begin{array}{c}2 n \\ n\end{array}\right) 312$ avoiding permutations in $\mathfrak{S}_{n}$, and gives us the following result [8, Cor. 7.5].

Proposition 5. Let $P$ be an n-element unit interval order and $w=w(P)$ the corresponding 312-avoiding permutation in $\mathfrak{S}_{n}$. Then we have $X_{\operatorname{inc}(P)}=Y\left(\widetilde{C}_{w}(1)\right)$.

Combinatorial interpretations of numbers $\theta(\operatorname{inc}(P))$ often involve structures called $P$-tableaux and statistics on these. Define a $P$-tableau of shape $\lambda \vdash|P|$ to be a filling of a (French) Young diagram of shape $\lambda$ with the elements of $P$, one per box. Given such a $P$-tableau $U$, let $U_{i}$ be the $i$ th row (from the bottom) of $U$, and let $U_{i, j}$ be the $j$ th entry in row $i$. If $P$-tableau $U$ consists of a single row, we will call it a $P$-permutation. In particular, each concatenation $U_{i_{1}} \circ \cdots \circ U_{i_{k}}$ of the rows (in any order) of a $P$-tableau $U$ is a $P$-permutation. If the elements of a poset are $[n]:=\{1, \ldots, n\}$, we will sometimes write a $P$-permutation as an ordinary permutation $v_{1} \cdots v_{n} \in \mathfrak{S}_{n}$. For example, (19) shows a poset $P$, a $P$-tableau $U$ of shape 32 , the second row $U_{2}$ and entry $U_{2,1}$ of $U$, and the $P$-permutation $U_{2} \circ U_{1}$ which may be viewed as an element of $\mathfrak{S}_{5}$.

$$
P=3
$$


The statistics we apply to $P$-tableaux are $P$-analogs of traditional permutation statistics. Call a position $(i, j)$ in $U$ a $P$-descent if $U_{i, j}>_{P} U_{i, j+1}$, and define $\operatorname{des}_{P}(U)$ to be the number of $P$ descents in $U$. Define $\bar{U}$ to be the $P$-tableau obtained from $U$ by ordering the elements in each row from least to greatest labels. That is, $\bar{U}_{i, j}$ is the entry of $U_{i}$ whose label is $j$ th-smallest, as an integer. Call a position $(i, j)$ in $U$ a $P$-excedance if $U_{i, j}>_{P} \bar{U}_{i, j}$, and define $\operatorname{exc}_{P}(U)$ to be the number of $P$-excedances in $U$. Call a position $(i, j)$ in $U$ a $P$-record if $U_{i, 1}, \ldots, U_{i, j-1}<_{P} U_{i, j}$, and call the record nontrivial if $j \neq 1$. Define $\operatorname{rec}_{P}(U)$ to be the number of nontrivial $P$-records in $U$. For example, we look again at the poset $P$ in (19), the $P$-tableaux there, and three more,

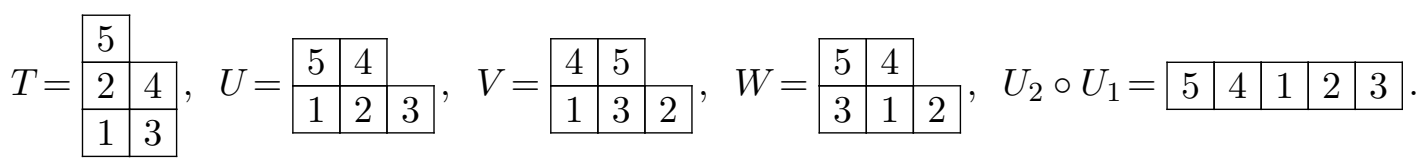

We have $\operatorname{des}_{P}(T)=\operatorname{des}_{P}(U)=\operatorname{des}_{P}(V)=0$, while $\operatorname{des}_{P}(W)=\operatorname{des}_{P}\left(U_{2} \circ U_{1}\right)=1$, because $3>_{P} 1$ and $4>_{P} 1$. We have that $\operatorname{rec}_{P}(U)=\operatorname{rec}_{P}\left(U_{2} \circ U_{1}\right)=\operatorname{rec}_{P}(W)=0$ since the first entry in each row of these tableaux is greater than or incomparable to the remaining entries in the same row. On the other hand, $\operatorname{rec}_{P}(T)=2$ since $1<_{P} 3$ and $2<_{P} 4$, and $\operatorname{rec}_{P}(V)=1$ since $1<_{P} 3$. Reordering the entries of each row in the tableaux above we obtain

$$
\bar{T}=T, \quad \bar{U}=\bar{V}=\bar{W}=\begin{array}{|l|l|l|l}
\hline 4 & 5 \\
\hline 1 & 2 & 3
\end{array}, \quad \overline{U_{2} \circ U_{1}}=\begin{array}{l|l|l|l|l|}
1 & 2 & 3 & 4 & 5
\end{array} .
$$

Comparing $T, U, V$ with $\bar{T}, \bar{U}, \bar{V}$, we have $\operatorname{exc}_{P}(T)=\operatorname{exc}_{P}(U)=\operatorname{exc}_{P}(V)=0$. Comparing $W$ and $\bar{W}$, we see that position $(1,1)$ is the only $P$-excedance: $3>_{P} 1$. $\operatorname{Thus} \operatorname{exc}_{P}(W)=1$. Comparing $U_{2} \circ U_{1}$ to $\overline{U_{2} \circ U_{1}}$, we see that positions $(1,1)$ and $(1,2)$ are both $P$-excedances: $5>_{P} 1,4>_{P} 2$. Thus $\operatorname{exc}_{P}\left(U_{2} \circ U_{1}\right)=2$.

Using comparability in $P$ and the above statistics, we define six classes of $P$-tableaux. Call a $P$-tableau $U$ of shape $\lambda$

1. P-descent-free or row-semistrict if $\operatorname{des}_{P}(U)=0$,

2. column-strict if the entries of each column satisfy $U_{i, j}<_{P} U_{i+1, j}$,

3. standard if it is column-strict and row-semistrict,

4. cyclically row-semistrict if it is row-semistrict and if $U_{i, \lambda_{i}} \ngtr_{P} U_{i, 1}$ for all $i$,

5. P-excedance-free if $\operatorname{exc}_{P}(U)=0$,

6. P-record-free if it has no nontrivial $P$-records.

For example, we may examine the tableaux in (20) for these properties to obtain the table

\begin{tabular}{|c|c|c|c|c|c|}
\hline & $T$ & $U$ & $V$ & $W$ & $U_{2} \circ U_{1}$ \\
\hline row-semistrict & $\checkmark$ & $\checkmark$ & $\checkmark$ & & \\
column-strict & & $\checkmark$ & $\checkmark$ & $\checkmark$ & $\checkmark$ \\
standard & & $\checkmark$ & $\checkmark$ & & \\
cyclically row-semistrict & & & $\checkmark$ & & \\
$P$-excedance-free & $\checkmark$ & $\checkmark$ & $\checkmark$ & & \\
$P$-record-free & & $\checkmark$ & & $\checkmark$ & $\checkmark$ \\
\hline
\end{tabular}

where the row-semistrict tableaux $T$ and $U$ fail to be cyclically row-semistrict because their first rows begin with 1 and end with $3>_{P} 1$. 


\subsection{Induced sign characters / monomial coefficients of $X_{\operatorname{inc}(P)}$}

By definition, the induced sign characters satisfy

$$
\begin{aligned}
\epsilon^{\lambda}(G) & =c(G, \lambda), \\
\epsilon^{\lambda}(\operatorname{inc}(P)) & =\# \text { column-strict } P \text {-tableaux of shape } \lambda^{\top}
\end{aligned}
$$

for all graphs $G=(V, E)$ and posets $P$. Since $\epsilon^{n}(G)=1$ if $G$ has no edges and is 0 otherwise, we can easily express $\epsilon^{\lambda}(G)$ in terms of subgraphs of $G$. For $J \subseteq V=[n]$, let $\bar{J}=[n] \backslash J$, and define

$$
\begin{aligned}
G_{J} & =\text { subgraph of } G \text { induced by vertices } J \\
P_{J} & =\text { subposet of } P \text { induced by elements } J .
\end{aligned}
$$

Given $\alpha=\left(\alpha_{1}, \ldots, \alpha_{r}\right) \vDash n$, call a sequence $\left(I_{1}, \ldots, I_{r}\right)$ of subsets of $[n]$ an ordered set partition of $[n]$ of type $\alpha$ if we have

1. $\left|I_{i}\right|=\alpha_{i}$ for $i=1, \ldots, r$,

2. $I_{i} \cap I_{j}=\varnothing$ for $i \neq j$,

3. $I_{1} \cup \cdots \cup I_{r}=[n]$.

Using (21) and the language of ordered set partitions, we can decompose some trace evaluations $\theta(G)$ as follows.

Lemma 6. Let $G$ be a graph on $n$ vertices.

1. If $\lambda=\left(\lambda_{1}, \ldots, \lambda_{r}\right) \vdash n$ is the weakly decreasing rearrangement of the parts of $\mu \vdash k$ and $\nu \vdash n-k$, then we have

$$
\epsilon^{\lambda}(G)=\sum_{\left(I_{1}, \ldots, I_{r}\right)} \epsilon^{\lambda_{1}}\left(G_{I_{1}}\right) \cdots \epsilon^{\lambda_{r}}\left(G_{I_{r}}\right)=\sum_{J \subseteq[n]} \epsilon^{\mu}\left(G_{J}\right) \epsilon^{\nu}\left(G_{\bar{J}}\right)
$$

where the first sum is over ordered set partitions of $[n]$ of type $\lambda$.

2. Let symmetric functions $t_{1} \in \Lambda_{k}, t_{2} \in \Lambda_{n-k}, t_{1} t_{2} \in \Lambda_{n}$ correspond by the Frobenius isomorphism to traces $\theta_{1} \in \mathcal{T}_{k}, \theta_{2} \in \mathcal{T}_{n-k}, \theta=\theta_{1} \otimes \theta_{2} \uparrow \mathfrak{S}_{\mathfrak{S}_{k} \times \mathfrak{S}_{n-k}} \in \mathcal{T}_{n}$. Then we have

$$
\theta(G)=\sum_{\substack{J \subseteq[n] \\|\bar{J}|=k}} \theta_{1}\left(G_{J}\right) \theta_{2}\left(G_{\bar{J}}\right)
$$

Proof. (1) $c(G, \lambda)$ equals the number of ordered set partitions $\left(I_{1}, \ldots, I_{r}\right)$ of $[n]$ of type $\lambda$ such that $G_{I_{j}}$ is an independent set for all $j$, and also the number of ordered set partitions of $[n]$ of type $\left(\mu_{1}, \ldots, \mu_{\ell(\mu)}, \nu_{1}, \ldots, \nu_{\ell(\nu)}\right)$ having the same property. 
(2) Express $t_{1}, t_{2}$ in the elementary bases of $\Lambda_{k}, \Lambda_{n-k}$ as

$$
t_{1}=\sum_{\mu \vdash k} a_{\mu} e_{\mu}, \quad t_{2}=\sum_{\nu \vdash k} b_{\nu} e_{\nu}
$$

and let $\lambda(\mu, \nu) \vdash n$ be the weakly decreasing rearrangement of the parts of $\mu$ and $\nu$. Then we have

$$
t_{1} t_{2}=\sum_{\substack{\mu \vdash k \\ \nu \vdash n-k}} a_{\mu} b_{\nu} e_{\lambda(\mu, \nu)}, \quad \theta(G)=\sum_{\substack{\mu \vdash k \\ \nu \vdash n-k}} a_{\mu} b_{\nu} \epsilon^{\lambda(\mu, \nu)}(G) .
$$

By $(22)$ and $(24), \theta(G)$ equals

$$
\sum_{\substack{\mu \vdash k \\ \nu \vdash n-k}} a_{\mu} b_{\nu} \sum_{\substack{J \subseteq[n] \\|\bar{J}|=k}} \epsilon^{\mu}\left(G_{J}\right) \epsilon^{\nu}\left(G_{\bar{J}}\right)=\sum_{\substack{J \subseteq[n] \\|\bar{J}|=k}} \sum_{\mu \vdash k} a_{\mu} \epsilon^{\mu}\left(G_{J}\right) \sum_{\nu \vdash n-k} b_{\nu} \epsilon^{\nu}\left(G_{\bar{J}}\right)=\sum_{\substack{J \subseteq[n] \\|\bar{J}|=k}} \theta_{1}\left(G_{J}\right) \theta_{2}\left(G_{\bar{J}}\right) .
$$

We will use this fact to prove similar formulas for induced trivial characters and power sum traces.

\subsection{Irreducible characters / Schur coefficients of $X_{\mathrm{inc}(P)}$}

While $\chi^{\lambda}(\operatorname{inc}(P))$ is negative for some posets $P$, Stanley and Stembridge [33, Conj. 5.1] conjectured it to be nonnegative for $(\mathbf{3}+\mathbf{1})$-free posets $P$. Gasharov [14] proved this by showing that for these posets we have

$$
\chi^{\lambda}(\operatorname{inc}(P))=\# \text { standard } P \text {-tableaux of shape } \lambda \text {. }
$$

Kaliszewski [18, Prop. 4.3] extended this result to all posets $P$ when $\lambda$ is a hook shape. We give an alternate proof of this fact using (21) and the inverse Kostka numbers, which satisfy

$$
\chi^{\mu}=\sum_{\lambda \vdash n} K_{\lambda, \mu^{\top}}^{-1} \epsilon^{\lambda} .
$$

For partitions $\lambda, \mu$ with $|\mu| \leqslant|\lambda|=n$ and $\mu_{i} \leqslant \lambda_{i}$ for all $i$, define a (skew) Young diagram of shape $\lambda / \mu$ to be the diagram obtained from a Young diagram of shape $\lambda$ by removing the $\mu_{i}$ leftmost boxes in row $i$ for all $i$. Call a Young diagram a border strip if it contains no $2 \times 2$ subdiagram of boxes. Define a special ribbon diagram of shape $\mu \vdash n$ and type $\lambda=\left(\lambda_{1}, \ldots, \lambda_{\ell}\right) \vdash n$ to be a Young diagram of shape $\mu$ subdivided into border strips (ribbons) of sizes $\lambda_{1}, \ldots, \lambda_{\ell}$, each of which contains a cell from the first row of $\mu$. Given a special ribbon diagram $Q$, define $\operatorname{sgn}(Q)$ to be -1 to the number of pairs of boxes in $Q$ which are horizontally adjacent and which belong to the same ribbon. It is known that we have

$$
K_{\lambda, \mu^{\top}}^{-1}=\sum_{Q} \operatorname{sgn}(Q)
$$

where the sum is over all special ribbon diagrams $Q$ of shape $\mu$ and type $\lambda$. (See $[2, \S 2]$.) For example, to expand $s_{3111}$ in the elementary basis, we draw a Young diagram of shape 
3111 and fill it with special ribbon diagrams

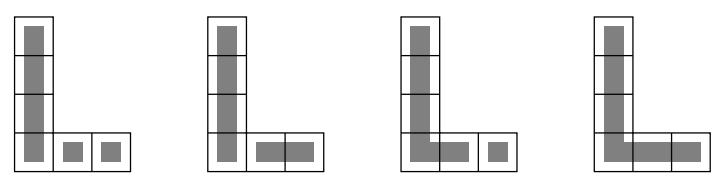

of types $411,42,51,6$, respectively, to obtain $s_{3111}=e_{411}-e_{42}-e_{51}+e_{6}$.

Special ribbon diagrams also relate column-strict $P$-tableaux of hook shape $\mu=$ $k 1^{n-k} \vdash n$ to column-strict $P$-tableaux of shape $\lambda \vdash n$ majorizing $\mu^{\top}$. To state this relationship precisely, we first observe that subsets of $[k-1]$ correspond bijectively to special ribbon diagrams of shape $\mu$. Let $D$ be a Young diagram of shape $\mu$. For each subset $S \subseteq[k-1]$, define $Q(\mu, S)$ to be the special ribbon diagram of shape $\mu$ whose ribbons are the equivalence classes defined by

1. $D_{i, 1} \sim D_{i+1,1}$ for $i=1, \ldots, n-k$,

2. $D_{1, j} \sim D_{1, j+1}$ for all $j \in S$,

and define $\lambda(\mu, S)$ to be the type of $Q(\mu, S)$. For example, when $\mu=31^{3}=(3,1,1,1)$ the ribbon diagrams in (28) correspond to the subsets $\varnothing,\{2\},\{1\}\{1,2\}$, respectively. This bijection leads to another.

Lemma 7. Fix hook partition $\mu=k 1^{n-k}$ and subset $S \subseteq[k-1]$, and define $Q=Q(\mu, S)$ and $\lambda=\lambda(\mu, S)$ as above. There is a bijection between column-strict $P$-tableaux $U$ of shape $\mu$ satisfying $U_{1, j}>_{P} U_{1, j+1}$ for all $j \in S$, and column-strict $P$-tableaux of shape $\lambda^{\top}$.

Proof. Let $\varphi$ be the claimed bijection. Given a column-strict $P$-tableau $U$ of shape $\mu$ satisfying $U_{1, j}>_{P} U_{1, j+1}$ for all $j \in S$, create $P$-tableau $\varphi(U)$ as follows.

1. Let $D$ be a Young diagram of shape $\lambda^{\top}$.

2. Label the ribbons of $Q$ from left to right as $1, \ldots, r$, and let $q_{i}$ be the number of boxes in ribbon $i$.

3. Superimpose $Q$ onto $U$.

4. For $i=1, \ldots, r$, place the elements of $U$ under ribbon $i$ into the leftmost unused column of $D$ which contains exactly $q_{i}$ boxes, so that elements strictly increase in the column from bottom to top.

The resulting $P$-tableau is clearly column-strict of shape $\lambda^{\top}$. To invert $\varphi$, suppose we are given a column-strict $P$-tableau $T$ of shape $\lambda^{\top}$.

1. Superimpose $Q$ onto an empty tableau $U$ of shape $\mu$.

2. For $i=1, \ldots, r$, fill $U$ by placing the elements from column $i$ of $T$ onto the leftmost available ribbon of $Q$ of length $\lambda_{i}^{\top}$ boxes, so that elements decrease from top to bottom, and from left to right. 
By the definition of $Q$, the resulting $P$-tableau $U$ has entries which satisfy the required inequalities, and it is easy to see that $\varphi(U)=T$.

As an example of the above bijection, fix $\mu=61, S=\{1,5\} \subseteq\{1,2,3,4,5\}$, and consider the pair

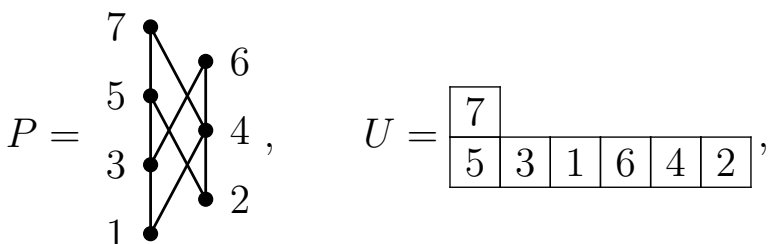

where $U$ is a column-strict $P$-tableau of shape $\mu$ which satisfies $U_{1, i}>_{P} U_{1, i+1}$, for $i=1,5$. Corresponding to $S$ is the special ribbon diagram

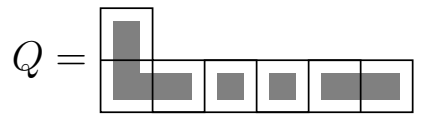

of shape $\mu$ and type 3211. Superimposing $Q$ onto $U$ and transforming ribbons into columns, we obtain the column-strict $P$-tableau

$$
\varphi(U)=\begin{array}{|l|l|l|l|}
\hline 7 & \multicolumn{3}{|l}{} \\
\hline 5 & 4 & \multicolumn{3}{|c}{} \\
\hline 3 & 2 & 1 & 6 \\
\hline
\end{array}
$$

of shape $3211^{\top}=421$

The above bijections allow for a new proof of Kaliszewski's result [18, Prop. 4.3].

Proposition 8. For any $n$-element poset $P$ and hook shape $k 1^{n-k} \vdash n$, the evaluation $\chi^{k 1^{n-k}}(\operatorname{inc}(P))$ equals the number of standard P-tableaux of shape $k 1^{n-k}$.

Proof. Fix $\mu=k 1^{n-k}$, let $a(\mu)$ be the number of standard $P$-tableaux of shape $\mu$, and for each subset $S \subseteq[k-1]$, let $b(\mu, S)$ be the number of column-strict $P$-tableaux $U$ of shape $\mu$ which satisfy

$$
U_{1, j}>_{P} U_{1, j+1} \quad \text { for all } j \in S .
$$

By the principle of inclusion-exclusion, these numbers are related by

$$
a(\mu)=\sum_{S \subseteq[k-1]}(-1)^{|S|} b(\mu, S) .
$$

Each subset $S \subseteq[k-1]$ corresponds to a special ribbon diagram $Q=Q(\mu, S)$ of shape $\mu$ as described before Lemma 7. The partition $\lambda(\mu, S)$ satisfies $|S|=k-\ell(\lambda(\mu, S))$.

By Lemma $7, b(\mu, S)$ is also the number of pairs $(Q, T)$ with $Q$ a special ribbon diagram of shape $\mu$ and type $\lambda(\mu, S)$, and $T$ a column-strict $P$-tableau of shape $\lambda^{\top}$. Thus we may rewrite (33) by summing over pairs $(S, U)$ and $(Q, T)$ satisfying the above conditions,

$$
a(\mu)=\sum_{(S, U)}(-1)^{|S|}=\sum_{(Q, T)}(-1)^{k-\ell(\operatorname{type}(Q))} .
$$


Now collect terms in the last sum which correspond to special ribbon diagrams sharing the same partition type $(Q) \vdash n$. Summing first over $\lambda \vdash n$ and then over special ribbon diagrams $Q$ of shape $\mu$ and type $\lambda$, we have that $a(\mu)$ is

$$
\sum_{\lambda \vdash n} \sum_{Q}(-1)^{k-\ell(\lambda)}\left(\# \text { column-strict } P \text {-tableaux of shape } \lambda^{\top}\right)=\sum_{\lambda \vdash n} \sum_{Q} \operatorname{sgn}(Q) \epsilon^{\lambda}(\operatorname{inc}(P)) \text {. }
$$

By (27) and (26), this is

$$
\sum_{\lambda \vdash n} K_{\lambda, \mu^{\top}}^{-1} \epsilon^{\lambda}(\operatorname{inc}(P))=\chi^{\mu}(\operatorname{inc}(P)) .
$$

\subsection{Induced trivial characters / monomial coefficients of $\omega X_{\operatorname{inc}(P)}$}

Given graph $G=(V, E)$ define an orientation of $G$ to be a directed graph $O=\left(V, E^{\prime}\right)$ obtained from $G$ by replacing each undirected edge $\{i, j\} \in E$ with exactly one of the directed edges $(i, j)$ or $(j, i)$. Call $O$ acyclic if it has no directed cycles. For example, a graph and two of its orientations are
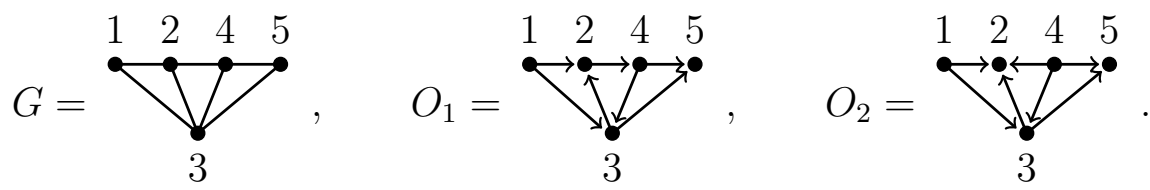

$\mathrm{O}_{2}$ is acyclic; $O_{1}$ is not.

By [30] we have for all graphs $G$ that

$$
\eta^{n}(G)=\# \text { acyclic orientations of } G \text {, }
$$

and as a consequence (or by Proposition 8) we have for all posets $P$ that

$$
\eta^{n}(\operatorname{inc}(P))=\# P \text {-descent-free } P \text {-permutations. }
$$

When $P$ is a unit interval order labeled as in (16) we also have [8, Thm. 4.7]

$$
\eta^{\lambda}(\operatorname{inc}(P))=\# P \text {-descent-free } P \text {-tableaux of shape } \lambda \text {. }
$$

We will extend this result to all posets in Theorem 10 and will include combinatorial interpretations related to $P$-excedance-free $P$-tableaux and acyclic orientations. To do so, we consider some straightforward extensions of permutation statistics to $P$-permutations.

Let $w$ be a $P$-permutation, and let $\operatorname{exc}_{P}(w)$ and $\operatorname{aexc}_{P}(w)$ be the numbers of $P$ excedances and $P$-antiexcedances in $w$,

$$
\operatorname{exc}_{P}(w)=\#\left\{i \mid w_{i}>_{P} i\right\}, \quad \operatorname{aexc}_{P}(w)=\#\left\{i \mid w_{i}<_{P} i\right\} .
$$

Let $\operatorname{des}_{P}(w)$ and $\operatorname{asc}_{P}(w)$ be the numbers of $P$-descents and $P$-ascents in $w$,

$$
\operatorname{des}_{P}(w)=\#\left\{i \mid w_{i}>_{P} w_{i+1}\right\}, \quad \operatorname{asc}_{P}(w)=\#\left\{i \mid w_{i}<_{P} w_{i+1}\right\} .
$$


Define the standard cycle notation of $w \in \mathfrak{S}_{n}$ to be the cycle notation in which cycles are listed in increasing order of their greatest elements, and these greatest elements are listed first in each cycle. Let $\sigma: \mathfrak{S}_{n} \rightarrow \mathfrak{S}_{n}$ be the bijection [31, §1.3] defined by setting $\sigma(w)$ equal to the permutation whose one-line notation is obtained by erasing parentheses from the standard cycle notation of $w$. For example, to compute $\sigma(5243761)$, we write 5243761 in standard cycle notation as $(2)(4,3)(6)(7,1,5)$, since the greatest elements of the cycles satisfy $2<4<6<7$. Then we erase parentheses to obtain 2436715 .

The following result is a strengthening of [31, Exercise 3.60c]. It first appeared with a different proof in [34, Thm.4.6].

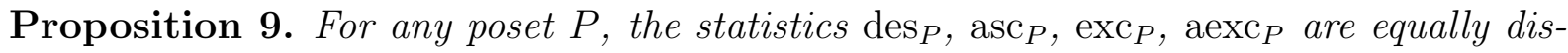
tributed on the set of all P-permutations.

Proof. $\left(\operatorname{des}_{P} \sim \operatorname{asc}_{P}\right)$ We have $\operatorname{asc}_{P}(w)=\operatorname{des}_{P}\left(w_{n} \cdots w_{1}\right)$.

$\left(\operatorname{exc}_{P} \sim \operatorname{aexc}_{P}\right)$ We have $\operatorname{aexc}_{P}(w)=\operatorname{exc}_{P}\left(w^{-1}\right)$.

$\left(\operatorname{des}_{P} \sim \operatorname{aexc}_{P}\right)$ Assume first that $P$ is naturally labeled (18). We claim that the map $\sigma$ satisfies $\operatorname{des}_{P}(\sigma(w))=\operatorname{aexc}_{P}(w)$. To see this, write $w$ in standard cycle notation and $\sigma(w)$ in one-line notation as

$$
\begin{aligned}
w & =\left(a_{1}, a_{2}, \ldots, a_{i_{1}}\right)\left(a_{i_{1}+1}, a_{i_{1}+2}, \ldots, a_{i_{2}}\right) \cdots\left(a_{i_{k-1}+1}, a_{i_{k-1}+2}, \ldots, a_{i_{k}}=a_{n}\right), \\
\sigma(w) & =a_{1}, a_{2}, \ldots, a_{i_{1}}, a_{i_{1}+1}, a_{i_{1}+2}, \ldots, a_{i_{2}}, \ldots, a_{i_{k-1}+1}, a_{i_{k-1}+2}, \ldots, a_{i_{k}}=a_{n} .
\end{aligned}
$$

Suppose that $j$ is a $P$-descent of $\sigma(w)$. By the natural labeling of $P$, we have $a_{j}>a_{j+1}$ and therefore $a_{j}$ can not appear last in its cycle in the standard cycle notation for $w$. Thus we have $a_{j}>_{P} a_{j+1}=w\left(a_{j}\right)$ and position $a_{j}$ is a $P$-antiexcedance of $w$. Thus position $a_{j}$ is a $P$-antiexcedance of $w$. Now suppose that $j$ is not a $P$-descent of $\sigma(w)$. Then in the standard cycle notation for $w$ we have either that $a_{j}, a_{j+1}$ appear consecutively in a cycle and satisfy $a_{j} \ngtr_{P} a_{j+1}=w\left(a_{j}\right)$, or that $a_{j}$ appears last in its cycle and satisfies $a_{j} \leqslant w\left(a_{j}\right)$. By the natural labeling of $P$, this last inequality implies $a_{j} \ngtr_{P} w\left(a_{j}\right)$. Thus in both cases position $a_{j}$ is not a $P$-antiexcedance of $w$.

Now assume that $P$ is nonnaturally labeled, and let $P^{\prime}$ be a naturally labeled copy of $P$. Then for some $u \in \mathfrak{S}_{n}$, the poset isomorphism $P \rightarrow P^{\prime}$ is given by $i \mapsto u_{i}$, and the bijection $w \mapsto u w$ from $\mathfrak{S}_{n}$ to itself satisfies $\operatorname{aexc}_{P}(w)=\operatorname{aexc}_{P^{\prime}}(u w)$.

Given a graph $G$ on $n$ vertices and an ordered set partition $\left(I_{1}, \ldots, I_{r}\right)$ of $[n]$ of type $\lambda \vdash n$, call the sequence $\left(G_{I_{1}}, \ldots, G_{I_{r}}\right)$ an ordered induced subgraph partition of $G$ of type $\lambda$. Let $\mathcal{I}_{\lambda}(G)$ be the set of such sequences, and define an acyclic orientation of an element of $\mathcal{I}_{\lambda}(G)$ to be a sequence $\left(O_{1}, \ldots, O_{r}\right)$, where $O_{j}$ is an acyclic orientation of $G_{I_{j}}$. For example, consider the ordered set partition $(234,15)$ of type $(3,2)$. A graph $G$, its ordered induced subgraph partition $\left(G_{234}, G_{15}\right)$, and one acyclic orientation $\left(O_{1}, O_{2}\right)$ of this are
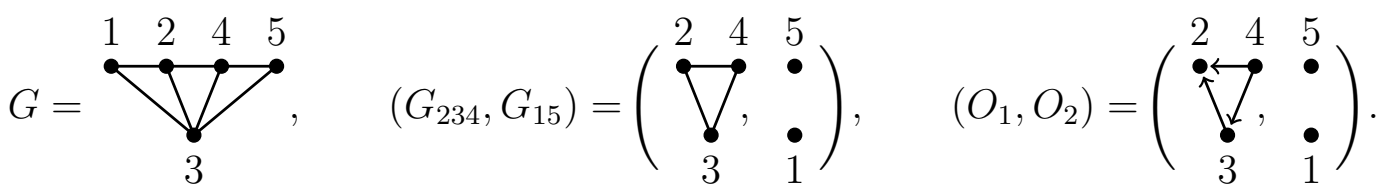
Theorem 10. For any poset $P$ and partition $\lambda=\left(\lambda_{1}, \ldots, \lambda_{r}\right) \vdash|P|$, the number $\eta^{\lambda}(\operatorname{inc}(P))$ has the combinatorial interpretations

1. \# P-descent-free P-tableaux of shape $\lambda$,

2. \# P-excedance-free P-tableaux of shape $\lambda$,

3. \# acyclic orientations of sequences $\left(\operatorname{inc}\left(P_{I_{1}}\right), \ldots, \operatorname{inc}\left(P_{I_{r}}\right)\right) \in \mathcal{I}_{\lambda}(\operatorname{inc}(P))$.

Proof. Let $\lambda=\left(\lambda_{1}, \ldots, \lambda_{r}\right)$. Since $h_{\lambda}=h_{\lambda_{1}} \cdots h_{\lambda_{r}}$, Lemma 6 implies that induced trivial characters satisfy

$$
\eta^{\lambda}(\operatorname{inc}(P))=\sum_{\left(I_{1}, \ldots, I_{r}\right)} \eta^{\lambda_{1}}\left(\operatorname{inc}\left(P_{I_{1}}\right)\right) \cdots \eta^{\lambda_{r}}\left(\operatorname{inc}\left(P_{I_{r}}\right)\right)
$$

where the sum is over all ordered set partitions of $|P|$ of type $\lambda$. By (35), we have for all $n$-element posets $P$ that $\eta^{n}(\operatorname{inc}(P))$ is the number of $P$-descent-free $P$-permutations. This implies interpretation (1) of the theorem, and then Proposition 9 implies interpretation (2).

Interpretation (3) follows from the known bijection between acyclic orientations of $\operatorname{inc}(P)$ and $P$-descent-free $P$-permutations. (See, e.g., [1, §4], [31, Exercise 3.60b].) Specifically, given an acyclic orientation $\left(O_{1}, \ldots, O_{r}\right)$ of a sequence $\left(\operatorname{inc}\left(P_{I_{1}}\right), \ldots, \operatorname{inc}\left(P_{I_{r}}\right)\right)$ in $\mathcal{I}_{\lambda}(\operatorname{inc}(P))$, create row-semistrict $P$-tableau $U$ of shape $\lambda$ as follows.

For $i=1, \ldots, r$, do

1. Initialize $U_{i}$ to be the empty Young diagram of shape $\lambda_{i}$.

2. While $O_{i}$ is not empty do

(a) Let $j$ be the minimum element of the chain in $P$ consisting of the source vertices in $O_{i}$.

(b) Update $O_{i}$ by removing $j$ and its outgoing edges.

(c) Update $U_{i}$ by placing $j$ in its leftmost empty box.

Given a row-semistrict $P$-tableau $U$ of shape $\lambda$, create an induced subgraph partition of inc $(P)$ and an acyclic orientation $\left(O_{1}, \ldots, O_{r}\right)$ of it as follows.

For $i=1, \ldots, r$, do

1. Let the vertices of $O_{i}$ be the set of elements in $U_{i}$.

2. For all pairs $(j, k)$ of entries of $U_{i}$ which are incomparable in $P$, if $j$ precedes $k$ in $U_{i}$, then create a directed edge from $j$ to $k$ in $O_{i}$.

The special case of Theorem 10 (2) corresponding to $P$ a unit interval order and $\lambda=n$ has an interpretation in terms of the Bruhat order on $\mathfrak{S}_{n}$. 
Corollary 11. Let $P$ be a unit interval order on $[n]$ labeled as in (16) and let $w \in \mathfrak{S}_{n}$ be the corresponding 312-avoiding permutation as in (17). Then we have

$$
\left\{v \in \mathfrak{S}_{n} \mid \operatorname{exc}_{P}(v)=0\right\}=\left\{v \in \mathfrak{S}_{n} \mid v \leqslant w\right\} .
$$

Proof. Define the matrix $A=\left(a_{i, j}\right)$ by

$$
a_{i, j}= \begin{cases}0 & \text { if } i<_{P} j \\ 1 & \text { otherwise }\end{cases}
$$

By [28, Lem. $5.3(3)]$, the product $a_{1, v_{1}} \cdots a_{n, v_{n}}$ is 1 if $v \leqslant w$ and is 0 otherwise. But $a_{1, v_{1}} \cdots a_{n, v_{n}}=1$ if and only if $i \nless_{P} v_{i}$ for $i=1, \ldots, n$, i.e., if and only if $v$ is $P$-excedance free.

\subsection{Power sum traces / scaled power sum coefficients of $\omega X_{\operatorname{inc}(P)}$}

It is known that we have $\psi^{\lambda}(\operatorname{inc}(P)) \geqslant 0$ for all $P$ [30], and

$$
\begin{aligned}
\psi^{\lambda}(\operatorname{inc}(P)) & =\# \text { cyclically row-semistrict } P \text {-tableaux of shape } \lambda \\
& =\# P \text {-record-free, row-semistrict } P \text {-tableaux of shape } \lambda
\end{aligned}
$$

for all unit interval orders $P$ labeled as in (16) [1, Thm.4], [8, Thm.4.7], [27, §7]. We will extend these results to all posets in Theorem 13, and will include more combinatorial interpretations involving inc $(P)$ and a related directed graph. Define $\operatorname{ngr}(P)$ to be the directed graph whose vertices are the elements of $P$ and whose edges are the ordered pairs $\left\{(i, j) \in P^{2} \mid i \ngtr_{P} j\right\}$, including loops $(i, i)$ for all $i \in P$. For example, a poset $P$ and related directed $\operatorname{graph} \operatorname{ngr}(P)$ are
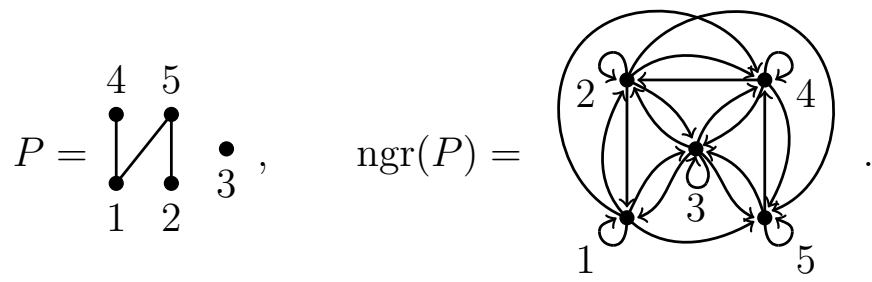

Given a directed graph $D$ on $n$ vertices and a partition $\lambda=\left(\lambda_{1}, \ldots, \lambda_{r}\right) \vdash n$, call a sequence $\left(H_{1}, \ldots, H_{r}\right)$ of vertex-disjoint subdigraphs of $D$ a disjoint cyclic vertex cover of $D$ of type $\lambda$ if $H_{j}$ is isomorphic to the cycle graph $C_{\lambda_{j}}$ for all $j$. For example, three disjoint cyclic vertex covers of the graph $\operatorname{ngr}(P)$ in $(40)$ are
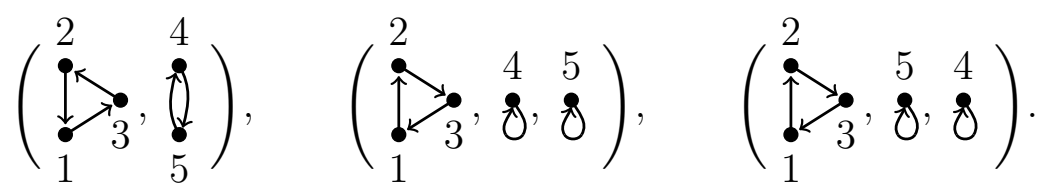

These have types 32 and 311. Observe that a collection of vertices does not completely specify a cycle, as this example shows two different cycles on the vertices $\{1,2,3\}$. 
To prove our results, we will use the transition matrix which relates the elementary and power sum bases of $\Lambda_{n}$. In particular,

$$
p_{n}=\sum_{\mu \vdash n}(-1)^{n-\ell(\mu)} c_{\mu} e_{\mu},
$$

where $c_{\mu}$ equals the number of subgraphs of the (labeled) cycle graph

$$
C_{n}=([n], E), \quad E=\{(i, i+1) \mid 1 \leqslant i \leqslant n-1\} \cup\{(n, 1)\},
$$

whose connected components are paths on $\mu_{1}, \ldots, \mu_{k}$ vertices. Clearly such subgraphs correspond bijectively to subsets $S \subseteq[n]$,

$$
S \longleftrightarrow C_{n, S}=\left([n], E_{S}\right) \text { with } E_{S}=\{(i, j) \in E \mid i \in S\}
$$

and we define $\mu(S)$ to be the weakly decreasing sequence of component cardinalities of $C_{n, S}$. We will also use the set of $P$-permutations whose (cyclic) $P$-descent set contains $S$,

$$
\mathcal{B}(S)=\left\{w \in \mathfrak{S}_{n} \mid w_{i}>_{P} w_{i+1}\left(\text { or } w_{i}=w_{n}>_{P} w_{1}\right) \text { for all } i \in S\right\} .
$$

Lemma 12. For any $n$-element poset $P$ and subset $S \subseteq[n]$, the permutations in $\mathcal{B}(S)$ correspond bijectively to column-strict P-tableaux of shape $\mu(S)$. In particular, we have $|\mathcal{B}(S)|=\epsilon^{\mu(S)}(\operatorname{inc}(P))$.

Proof. Fix $w \in \mathcal{B}(S)$. For each maximal interval $[i, j](\bmod n)$ with $i, \ldots, j \in S$, we have the chain $w_{i}>_{P} \cdots>_{P} w_{j+1}$; for $i-1, i \notin S$, we have the one-element chain $w_{i}$. Let $\mu=\mu(S)$ and insert the $\ell(\mu)=n-|S|$ chains into the columns of a Young diagram of shape $\mu^{\top}$ to obtain a column-strict $P$-tableau. For chains of equal cardinalities, fill the leftmost available column of the tableau with the leftmost available chain in $w$ (considering $\cdots>_{P} w_{n}>_{P} w_{1}>_{P} \cdots$ to be the leftmost chain of all, if it exists). It is easy to see that this map is invertible.

As an example of the above bijection, consider the poset, subset, and $P$-permutation

$$
P={ }_{1}^{4}, \quad S=\{1,4,5,7\}, \quad w=5316427
$$

with $w \in \mathcal{B}(\{1,4,5,7\})$ because the cyclic $P$-descents of $w$ include $1,4,5,7$ :

$$
w_{1}>_{P} w_{2}, \quad w_{4}>_{P} w_{5}, \quad w_{5}>_{P} w_{6}, \quad w_{7}>_{P} w_{1} .
$$

Combining these $P$-descents to form chains (and collecting leftover 1-chains), we have

$$
w_{7}>_{P} w_{1}>_{P} w_{2}=753, \quad w_{3}=1, \quad w_{4}>_{P} w_{5}>_{P} w_{6}=642,
$$


which we can insert in order of weakly decreasing cardinality into a column-strict $P$ tableau of shape $331^{\top}=322$

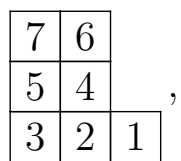

where we have broken the tie between 3-element chains by inserting the leftmost chain (the one containing $w_{1}$ ) first.

Now we may interpret $\psi^{\lambda}(\operatorname{inc}(P))$ as follows.

Theorem 13. For any poset $P$ and partition $\lambda=\left(\lambda_{1}, \ldots, \lambda_{r}\right) \vdash|P|$, the number $\psi^{\lambda}(\operatorname{inc}(P))$ has the combinatorial interpretations

1. \# cyclically row-semistrict $P$-tableaux of shape $\lambda$,

2. \# P-record-free, row-semistrict P-tableaux of shape $\lambda$,

3. \# disjoint cyclic vertex covers of $\operatorname{ngr}(P)$ of type $\lambda$,

4. \# acyclic orientations $\left(O_{1}, \ldots, O_{r}\right)$ of sequences $\left(\operatorname{inc}\left(P_{I_{1}}\right), \ldots, \operatorname{inc}\left(P_{I_{r}}\right)\right) \in \mathcal{I}_{\lambda}(\operatorname{inc}(P))$ in which each oriented component $O_{i}$ has exactly one source.

Proof. Since $p_{\lambda}=p_{\lambda_{1}} \cdots p_{\lambda_{r}}$, Lemma 6 implies that power sum traces satisfy

$$
\psi^{\lambda}(\operatorname{inc}(P))=\sum_{\left(I_{1}, \ldots, I_{r}\right)} \psi^{\lambda_{1}}\left(\operatorname{inc}\left(P_{I_{1}}\right)\right) \cdots \psi^{\lambda_{r}}\left(\operatorname{inc}\left(P_{I_{r}}\right)\right),
$$

where the sum is over all ordered set partitions of $|P|$ of type $\lambda$.

(1) We claim that for any $n$-element poset $P, \psi^{n}(\operatorname{inc}(P))$ is the number of cyclically rowsemistrict $P$-permutations $\left(P\right.$-descent-free $w_{1} \cdots w_{n}$ with $\left.w_{n} \ngtr_{P} w_{1}\right)$. To see this, let $a$ be the number of such $P$-permutations and define $\mathcal{B}(S)$ as in (42). By the principle of inclusion/exclusion and Lemma 12, the cardinalities $a$ and $|\mathcal{B}(S)|$ are related by

$$
a=\sum_{S \subseteq[n]}(-1)^{|S|}|\mathcal{B}(S)|=\sum_{S \subseteq[n]}(-1)^{|n-\ell(\mu(S))|} \epsilon^{\mu(S)}(\operatorname{inc}(P)) .
$$

By (41) the number of distinct subsets $T \subseteq[n]$ satisfying $\mu(T)=\mu(S)$ is $c_{\mu(S)}$. Therefore we have

$$
a=\sum_{\mu \vdash n}(-1)^{n-\ell(\mu)} c_{\mu} \epsilon^{\mu}(\operatorname{inc}(P))=\psi^{n}(\operatorname{inc}(P)) \text {, }
$$

as desired.

(2) Stanley [30, Thm. 3.3] showed that $\phi^{n}(P)=\psi^{n}(P)$ equals the number of acyclic orientations of inc $(P)$ having exactly one sink. Reversing all edges in such an orientation and applying the bijection at the end of the proof of Theorem 10, we have that $\psi^{n}(P)$ equals the number of $P$-record-free $P$-descent-free $P$-permutations. Then by $(44)$ we have the desired result. 
(3) Let $U$ be a cyclically row-semistrict $P$-tableau of shape $\lambda$. Each pair of horizontally adjacent entries $\left(U_{i, j}, U_{i, j+1}\right)$ (or $\left.\left(U_{i, \lambda_{i}}, U_{i, 1}\right)\right)$ in $U$ corresponds to an edge in $\operatorname{ngr}(P)$, and the row $U_{i}$ corresponds to a cycle. Removing all other edges from $\operatorname{ngr}(P)$ and listing the cycles in order of their corresponding rows of $U$, we obtain the desired disjoint cyclic vertex cover.

(4) As described above, $\psi^{n}(P)$ equals the number of acyclic orientations of inc $(P)$ having exactly one source. Now (44) gives the desired result.

\subsection{Monomial traces / elementary coefficients of $X_{\operatorname{inc}(P)}$}

While $\phi^{\lambda}(\operatorname{inc}(P))$ is negative for some posets $P$, Stembridge and Stanley conjectured [33, Conj. 5.5] that for $(\mathbf{3}+\mathbf{1})$-free posets $P$ we have

$$
\phi^{\lambda}(\operatorname{inc}(P)) \geqslant 0 \text {. }
$$

By [15, Thm. 5.1], this conjecture is equivalent to the assertion that (45) holds when $P$ is a unit interval order. Using this fact, we may state special cases of the conjecture which are known to be true. In particular, the following conditions on $\lambda$ and/or $P$ imply (45).

1. P 3-free [15, Thm. 5.3], [30, Cor. 3.6].

2. $P$ a 4-free unit interval order [6, Thm. 1.8].

3. $\lambda$ a rectangular partition, i.e., $\lambda_{1}=\lambda_{2}=\cdots=k$, and $P(\mathbf{3}+\mathbf{1})$-free [36, Thm. 2.8].

4. $P$ a unit interval order with a $\left(\lambda_{1}+1\right)$-element antichain [8, Thm. 3.7, Prop. 10.1].

5. $P$ a unit interval order with component sizes of inc $(P)$ not refining $\lambda$ [8, Prop. 10.2].

6. $\lambda_{1} \leqslant 2$ and $P(\mathbf{3}+\mathbf{1})$-free [8, Thm. 10.3].

7. $P$ defined on $[n]$ by $i<_{P} j$ if $i+1<j$ (as integers) [5, p. 242], [30, Prop. 5.3].

8. $P$ defined on $[n]$ by $i<_{P} j$ if $i+2<j$ (as integers) [12].

9. $P$ defined on $[n]$ by $i<_{P} j$ if $i+n-3<j$ (as integers) [27].

Conditions (4) and (5) above more specifically imply that we have $\phi^{\lambda}(\operatorname{inc}(P))=0$. Another related result concerns sums of monomial traces [30, Thm.3.3].

Proposition 14. For all graphs $G$ on $n$ vertices we have

$$
\sum_{\substack{\lambda \vdash n \\ \ell(\lambda)=k}} \phi^{\lambda}(G)=\text { \# acyclic orientations of } G \text { having } k \text { sources, }
$$

and for all $n$-element posets $P$ we have

$$
\sum_{\substack{\lambda \vdash n \\ \ell(\lambda)=k}} \phi^{\lambda}(\operatorname{inc}(P))=\# P \text {-descent-free P-permutations w with } k \text { P-records. }
$$


Since $\phi^{1^{n}}=\epsilon^{n}$ and $\phi^{n}=\psi^{n}$, it is tempting to conjecture a formula for $\phi^{\lambda}(\operatorname{inc}(P))$ which combines column-strictness of Equation (21) with one of the conditions of Theorem 13. Two obvious combinations do not in general give correct formulas. For example, the following poset $P$ and monomial trace evaluations

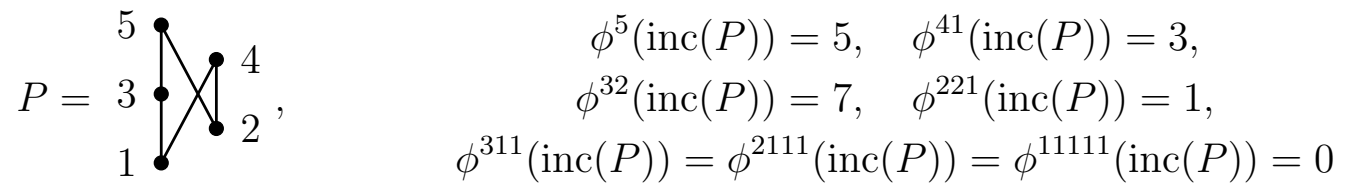

are not consistent with the number of standard, cyclically row-semistrict $P$-tableaux of shape 32

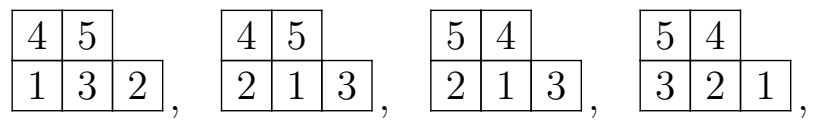

or the number of standard, $P$-record-free $P$-tableaux of shape 32

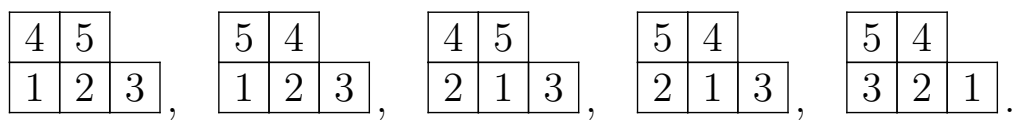

The author has found that for $n \leqslant 5$, the sets of analogous tableaux for $n$-element posets have cardinalities no greater than the true values of $\phi^{\lambda}(\operatorname{inc}(P))$. This suggests the following question.

Question 15. Do we have for all unit interval orders $P$ and all partitions $\lambda \vdash|P|$, that $\phi^{\lambda}(\operatorname{inc}(P))$ is greater than or equal to

1. the number of standard, cyclically row-semistrict $P$-tableaux of shape $\lambda$ ?

2. the number of standard, $P$-record-free $P$-tableaux of shape $\lambda$ ?

\subsection{Fundamental expansion of $X_{\text {inc }(P)}$}

We remark that for any graph $G$, there are known combinatorial interpretations for the coefficients arising in the fundamental expansions of $X_{G}$ and $\omega X_{G}$. These are easiest to express in the special case that $G$ is the incomparability graph of an $n$-element poset $P$. Writing

$$
\begin{aligned}
X_{\operatorname{inc}(P)} & =\sum_{S \subseteq[n-1]} \xi^{S}(\operatorname{inc}(P)) F_{n,[n-1] \backslash S}, \\
\omega X_{\operatorname{inc}(P)} & =\sum_{S \subseteq[n-1]} \xi^{S}(\operatorname{inc}(P)) F_{n, S},
\end{aligned}
$$

we have that $\xi^{S}(\operatorname{inc}(P))$ is the number of $P$-permutations with $P$-descent set $S$ [7, Cor. 2]. For combinatorial interpretations corresponding to an arbitrary graph $G$, see [7, Cor. 1]. 


\subsection{Trace identities}

Symmetric function identities, Lemma 6, and the combinatorial interpretations stated in Equation (21) - Theorem 13 lead to some identities relating $P$-permutations to pairs of subposet permutations. For instance, the first identity in the following result implies that the number of ways to create a row-semistrict $P$-permutation and circle one element equals the number of $P$-permutations $w_{1} \cdots w_{n}$ with $w_{1} \cdots w_{i}$ a cyclically row-semistrict $P_{J}$-permutation for some $i$-element set $J \subseteq[n](0<i \leqslant n)$, and $w_{i+1} \cdots w_{n}$ a rowsemistrict $P_{\bar{J}}$-permutation.

Corollary 16. Let $G$ be a graph on $n$ vertices. We have

$$
\begin{gathered}
n \eta^{n}(G)=\sum_{i=1}^{n} \sum_{\substack{J \subseteq[n] \\
|\bar{J}|=i}} \psi^{i}\left(G_{J}\right) \eta^{n-i}\left(G_{\bar{J}}\right), \\
n \epsilon^{n}(G)=\sum_{i=1}^{n} \sum_{\substack{J \subseteq[n] \\
|J|=i}}(-1)^{i-1} \psi^{i}\left(G_{J}\right) \epsilon^{n-i}\left(G_{\bar{J}}\right), \\
\sum_{i=0}^{n} \sum_{\substack{J \subseteq[n] \\
|J|=i}}(-1)^{i} \epsilon^{i}\left(G_{J}\right) \eta^{n-i}\left(G_{\bar{J}}\right)=0 .
\end{gathered}
$$

Proof. Applying Lemma 6 (2) to the symmetric function identities

$$
n h_{n}=\sum_{i=1}^{n} p_{i} h_{n-i}, \quad n e_{n}=\sum_{i=1}^{n}(-1)^{i-1} p_{i} e_{n-i}, \quad \sum_{i=0}^{n} e_{i} h_{n-i}=0,
$$

we obtain the claimed graph identities.

\section{Applications to total nonnegativity}

Nonnegative expansions of chromatic symmetric functions in the standard bases are closely related to functions of totally nonnegative matrices. We will make this relationship precise in Corollary 23.

Call a real $n \times n$ matrix $A=\left(a_{i, j}\right)$ totally nonnegative if for each pair $(I, J)$ of subsets of $[n]$, the square submatrix $A_{I, J}:=\left(a_{i, j}\right)_{i \in I, j \in J}$ satisfies $\operatorname{det}\left(A_{I, J}\right) \geqslant 0$. Such matrices are closely related to directed graphs called planar networks. Define a (nonnegative weighted) planar network of order $n$ to be a directed, planar, acyclic digraph $D=(V, E)$ which can be embedded in a disc so that $2 n$ distinguished vertices labeled clockwise as $s_{1}, \ldots, s_{n}, t_{n}, \ldots, t_{1}$ lie on the boundary of the disc, with a nonnegative real weight $c_{u, v}$ assigned to each edge $(u, v) \in E$. We may assume that $s_{1}, \ldots, s_{n}$, called sources, have indegree 0 and that $t_{n}, \ldots, t_{1}$, called sinks, have outdegree 0 . To every source-to-sink path, we associate a weight equal to the product of weights of its edges, and we define 
the path matrix $A=A(D)=\left(a_{i, j}\right)_{i, j \in[n]}$ by setting $a_{i, j}$ equal to the sum of weights of all paths from $s_{i}$ to $t_{j}$. For example, we have the following planar network $D$ of order 3 , in which unlabeled edges have weight 1 , and its path matrix $A$.

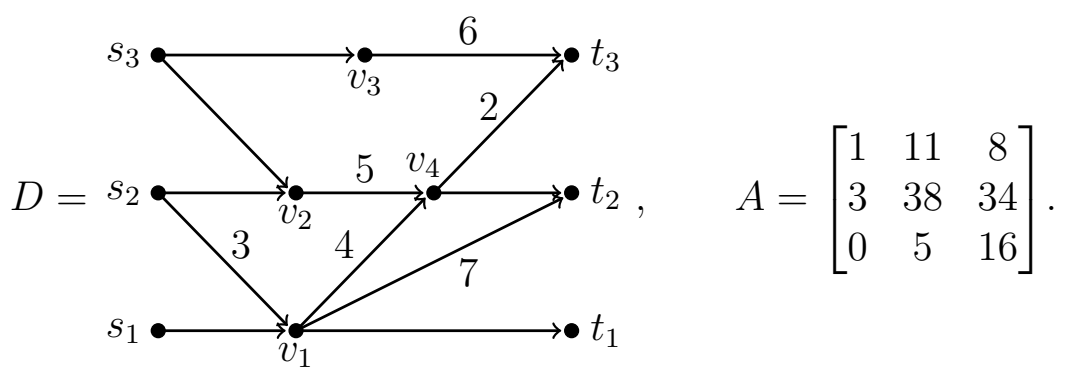

A result often attributed to Lindström [21] but proved earlier by Karlin and McGregor [19] asserts the total nonnegativity of such a matrix.

Theorem 17. The path matrix A of a nonnegative weighted planar network $D$ of order $n$ is totally nonnegative. Moreover, the nonnegative number $\operatorname{det}(A)$ equals

$$
\sum_{\pi} \operatorname{wgt}(\pi)
$$

where the sum is over all families $\pi=\left(\pi_{1}, \ldots, \pi_{n}\right)$ of pairwise nonintersecting paths in $D$, with $\pi_{i}$ a path from $s_{i}$ to $t_{i}$ for $i=1, \ldots, n$, and where

$$
\operatorname{wgt}(\pi):=\operatorname{wgt}\left(\pi_{1}\right) \cdots \operatorname{wgt}\left(\pi_{n}\right) .
$$

Since each submatrix of a totally nonnegative matrix is itself totally nonnegative, this result gives a combinatorial interpretation of the nonnegative numbers $\operatorname{det}\left(A_{I, J}\right)$ as well: $\operatorname{det}\left(A_{I, J}\right)$ is the sum of weights of all nonintersecting path families in $D$ from sources indexed by $I$ to sinks indexed by $J$ (assuming $|I|=|J|$ ). For example, in (46), we have $\operatorname{det}(A)=30$, and the one nonintersecting path family in $D$ from $\left\{s_{1}, s_{2}, s_{3}\right\}$ to $\left\{t_{1}, t_{2}, t_{3}\right\}$ has weight $(1 \cdot 1)(1 \cdot 5 \cdot 1)(1 \cdot 6)=30$. Also, we have $\operatorname{det}\left(A_{13,23}\right)=136$ and the three nonintersecting path families from $\left\{s_{1}, s_{3}\right\}$ to $\left\{t_{2}, t_{3}\right\}$ have weights $(1 \cdot 7)(1 \cdot 5 \cdot 2),(1 \cdot 7)(1 \cdot 6)$, and $(1 \cdot 4 \cdot 1)(1 \cdot 6)$, which sum to 136 .

The converse of Theorem 17 is true as well. That is, path matrices are essentially the only examples of totally nonnegative matrices [4], [11], [23], [37].

Theorem 18. For each $n \times n$ totally nonnegative matrix A, there exists a nonnegative weighted planar network $D$ of order $n$ whose path matrix is $A$.

Generalizing the determinant are matrix functions $\operatorname{Imm}_{\theta}: \operatorname{Mat}_{n \times n}(\mathbb{C}) \rightarrow \mathbb{C}$ called immanants and parametrized by linear functionals $\theta: \mathbb{C}\left[\mathfrak{S}_{n}\right] \rightarrow \mathbb{C}$. Define

$$
\operatorname{Imm}_{\theta}(A)=\sum_{w \in \mathfrak{S}_{n}} \theta(w) a_{1, w_{1}} \cdots a_{n, w_{n}}
$$


In the language of Section 2, we have $\operatorname{det}(A)=\operatorname{Imm}_{\epsilon^{n}}(A)$. For some functions $\theta$, the number $\operatorname{Imm}_{\theta}(A)$ is nonnegative for all totally nonnegative matrices $A$ and has a nice combinatorial interpretation in terms of families of paths in $D$. We say that a path family $\pi=\left(\pi_{1}, \ldots, \pi_{n}\right)$ in a planar network $D$ has type $w=w_{1} \cdots w_{n} \in \mathfrak{S}_{n}$ if for $i=1, \ldots, n$, the path $\pi_{i}$ begins at the source $s_{i}$ and terminates at the sink $t_{w_{i}}$. Define the sets

$$
\begin{aligned}
\mathcal{P}_{w}(D) & =\{\pi \text { in } D \mid \operatorname{type}(\pi)=w\} \\
\mathcal{P}(D) & =\bigcup_{w \in \mathfrak{S}_{n}} \mathcal{P}_{w}(D)
\end{aligned}
$$

Each path family $\pi=\left(\pi_{1}, \ldots, \pi_{n}\right) \in \mathcal{P}_{w}(D)$ forms a poset $P=P(\pi)$ defined by $\pi_{i}<_{P} \pi_{j}$ if $i<j$ (as integers) and $\pi_{i}$ does not intersect $\pi_{j}$. For example, the planar network $D$ in (46) has two path families of type 132. These and their posets are

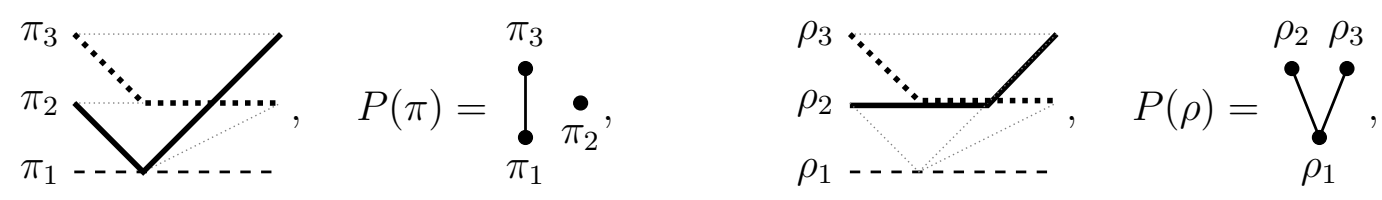

so $\mathcal{P}_{132}(D)=\{\pi, \rho\}$.

Observe that if path families $\pi, \sigma$ in $D$ consist of the same multiset $K$ of edges of $D$, then they satisfy $\operatorname{wgt}(\pi)=\operatorname{wgt}(\sigma)$. Call such a multiset $K$ a bijective skeleton, and define $\operatorname{wgt}(K)$ to be the product of its edge weights, with multiplicities. Define the sets

$$
\begin{aligned}
\Pi(K) & =\{\pi \in \mathcal{P}(D) \mid \text { edge multiset of } \pi \text { is } K\}, \\
\Pi_{w}(K) & =\{\pi \in \Pi(K) \mid \text { type }(\pi)=w\},
\end{aligned}
$$

and the $\mathbb{Z}\left[\mathfrak{S}_{n}\right]$-element

$$
z(K)=\sum_{\pi \in \Pi(K)} \operatorname{type}(\pi)
$$

For example, let bijective skeleton $K$ be the multiset of edges of $D(46)$ covered the path family $\rho$ in (48), and let $\sigma$ be the unique path family of type 123 covering $K$. Then we have
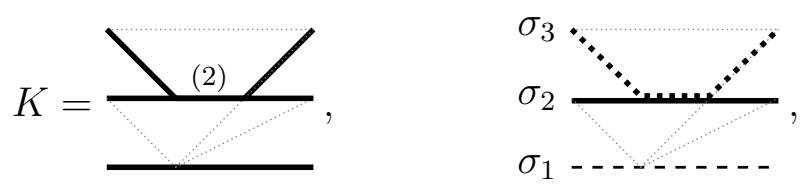

with (2) marking the edge in $K$ having multiplicity 2 . We also have

$$
\begin{gathered}
\Pi_{132}(K)=\{\rho\}, \quad \Pi_{123}(K)=\{\sigma\}, \quad \Pi(K)=\{\rho, \sigma\}, \\
\operatorname{wgt}(K)=\operatorname{wgt}(\rho)=\operatorname{wgt}(\sigma)=50, \quad z(K)=132+123=\widetilde{C}_{132}(1) .
\end{gathered}
$$

It is known that for any bijective skeleton $K, z(K)$ equals a product of Kazhdan-Lusztig basis elements $\widetilde{C}_{w}(1) \in \mathbb{Z}\left[\mathfrak{S}_{n}\right]$ indexed by 312 -avoiding permutations, and that for any totally nonnegative matrix $A$, the numbers $\operatorname{Imm}_{\theta}(A)$ and $\theta(z(K))$ are closely related. (See, e.g., [35, Thm. 2.1].) 
Proposition 19. Let $A$ be the path matrix of a weighted planar network $D$. Then for any linear functional $\theta: \mathbb{C}\left[\mathfrak{S}_{n}\right] \rightarrow \mathbb{C}$, we have

$$
\operatorname{Imm}_{\theta}(A)=\sum_{K} \operatorname{wgt}(K) \theta(z(K)),
$$

where the sum is over all bijective skeletons $K$ in $D$.

Proof. By the definition of path matrix, we can interpret each product of matrix entries appearing in $\operatorname{Imm}_{\theta}(A)$ as

$$
a_{1, w_{1}} \cdots a_{n, w_{n}}=\sum_{K} \sum_{\pi \in \Pi_{w}(K)} \operatorname{wgt}(\pi)=\sum_{K} \operatorname{wgt}(K)\left|\Pi_{w}(K)\right| .
$$

Multiplying each product by $\theta(w)$, summing over $w \in \mathfrak{S}_{n}$, and using the linearity of $\theta$, we may thus express $\operatorname{Imm}_{\theta}(A)$ as

$$
\begin{aligned}
\sum_{w \in \mathfrak{S}_{n}} \theta(w) \sum_{K} \operatorname{wgt}(K)\left|\Pi_{w}(K)\right| & =\sum_{K} \operatorname{wgt}(K) \sum_{w \in \mathfrak{S}_{n}} \theta(w)\left|\Pi_{w}(K)\right| \\
& =\sum_{K} \operatorname{wgt}(K) \theta\left(\sum_{w \in \mathfrak{S}_{n}}\left|\Pi_{w}(K)\right| w\right) \\
& =\sum_{K} \operatorname{wgt}(K) \theta\left(\sum_{\pi \in \Pi(K)} \operatorname{type}(w)\right) .
\end{aligned}
$$

Sometimes a combinatorial interpretation for $\operatorname{Imm}_{\theta}(A)$ comes from careful consideration of $\theta(z(K))$; other times it comes from a simple expression for $\operatorname{Imm}_{\theta}(A)$, such as the Littlewood-Merris-Watkins identities [22, §6.5], [25, §1],

$$
\begin{aligned}
& \operatorname{Imm}_{\epsilon^{\lambda}}(A)=\sum_{\left(I_{1}, \ldots, I_{r}\right)} \operatorname{det}\left(A_{I_{1}, I_{1}}\right) \cdots \operatorname{det}\left(A_{I_{r}, I_{r}}\right), \\
& \operatorname{Imm}_{\eta^{\lambda}}(A)=\sum_{\left(I_{1}, \ldots, I_{r}\right)} \operatorname{per}\left(A_{I_{1}, I_{1}}\right) \cdots \operatorname{per}\left(A_{I_{r}, I_{r}}\right),
\end{aligned}
$$

where the sums are over ordered set partitions of $[n]$ of type $\lambda=\left(\lambda_{1}, \ldots, \lambda_{r}\right)$.

\subsection{Induced sign character immanants}

Combinatorial interpretations for the immanants $\operatorname{Imm}_{\epsilon^{\lambda}}(A)$ follow easily from Theorem 17 and (49).

Theorem 20. Let planar network $D$ have path matrix A. Then we have

$$
\operatorname{Imm}_{\epsilon^{\lambda}}(A)=\sum_{K} \operatorname{wgt}(K) \sum_{\pi \in \Pi_{e}(K)} \epsilon^{\lambda}(\operatorname{inc}(P(\pi))) .
$$


Proof. By Theorem 17 and the comment immediately following it, the term of (49) corresonding to a fixed ordered set partition $\left(I_{1}, \ldots, I_{r}\right)$ is equal to the sum of weights of path families $\pi=\left(\pi_{1}, \ldots, \pi_{n}\right)$ of type $e$ in which for $j=1, \ldots, r$, paths indexed by $I_{j}$ are pairwise nonintersecting. This partitioned path family naturally forms a column-strict tableau $U=U\left(\pi, I_{1}, \ldots, I_{r}\right)$ of shape $\lambda^{\top}$, if we place paths indexed by $I_{j}$ into column $j$. We may therefore write the right-hand-side of (49) as

$$
\begin{aligned}
\sum_{K} \operatorname{wgt}(K) \sum_{\pi \in \Pi_{e}(K)} \#\left\{\left(I_{1}, \ldots, I_{r}\right) \mid U\left(\pi, I_{1}, \ldots, I_{r}\right) \text { is column-strict of shape } \lambda^{\top}\right\} \\
=\sum_{K} \operatorname{wgt}(K) \sum_{\pi \in \Pi_{e}(K)} \# \text { column-strict } P(\pi) \text {-tableaux of shape } \lambda^{\top} \\
=\sum_{K} \operatorname{wgt}(K) \sum_{\pi \in \Pi_{e}(K)} \epsilon^{\lambda}(\operatorname{inc}(P(\pi))) .
\end{aligned}
$$

Combining Proposition 19 with Theorem 20, we find that the inner sum of (51) is $\epsilon^{\lambda}(z(K))$ and we generalize this fact as follows.

Corollary 21. Let $K$ be a bijective skeleton in a planar network $D$. Then for all traces $\theta: \mathbb{C}\left[\mathfrak{S}_{n}\right] \rightarrow \mathbb{C}$, we have

$$
\theta(z(K))=\sum_{\pi \in \Pi_{e}(K)} \theta(\operatorname{inc}(P(\pi)))
$$

Proof. Weight the planar network $D$ by algebraically independent real numbers and let $A$ be its path matrix. By Proposition 19, we have

$$
\operatorname{Imm}_{\epsilon^{\mu}}(A)=\sum_{K} \operatorname{wgt}(K) \epsilon^{\mu}(z(K))
$$

where the sum is over all bijective skeletons $K$ in $D$. Since the edge weights of $D$ are algebraically independent, we may compare this expression to the right-hand-side of (51) to obtain

$$
\epsilon^{\mu}(z(K))=\sum_{\pi \in \Pi_{e}(K)} \epsilon^{\mu}(\operatorname{inc}(P(\pi)))
$$

Expanding $\theta$ in the induced sign character basis $\left\{\epsilon^{\mu} \mid \mu \vdash n\right\}$, we obtain the desired result.

Proposition 19 and Corollary 21 show that for $\theta \in \mathcal{T}_{n}$ we may compute $\operatorname{Imm}_{\theta}(A)$ by considering a planar network $D$ having path matrix $A$, each path family $\pi$ of type $e$ in $D$, and the corresponding chromatic symmetric function $X_{\operatorname{inc}(P(\pi))}$.

Corollary 22. For D a planar network having path matrix A, we have

$$
\operatorname{Imm}_{\theta}(A)=\sum_{K} \operatorname{wgt}(K) \sum_{\pi \in \Pi_{e}(K)} \theta(\operatorname{inc}(P(\pi))
$$

where $K$ varies over all bijective skeletons in $D$. 
Thus if $\theta \in \mathcal{T}_{n}$ satisfies $\theta(\operatorname{inc}(P)) \geqslant 0$ for all posets $P$, then it also satisfies $\operatorname{Imm}_{\theta}(A) \geqslant$ 0 for all totally nonnegative matrices $A$. For the convenience of the reader we summarize this and other known implications as follows.

Corollary 23. For $\theta \in \mathcal{T}_{n}$, the statements

1. $\theta\left(\widetilde{C}_{w}(1)\right) \geqslant 0$ for all permutations $w \in \mathfrak{S}_{n}$,

2. $\theta\left(\widetilde{C}_{w^{(1)}}(1) \cdots \widetilde{C}_{w^{(k)}}(1)\right) \geqslant 0$ for all sequences $\left(w^{(1)}, \ldots, w^{(k)}\right)$ of maximal elements of parabolic subgroups of $\mathfrak{S}_{n}$,

3. $\theta(\operatorname{inc}(P)) \geqslant 0$ for all posets $P$,

4. $\operatorname{Imm}_{\theta}(A) \geqslant 0$ for all totally nonnegative matrices $A$,

5. $\theta(\operatorname{inc}(P)) \geqslant 0$ for all unit interval orders $P$,

6. $\theta(\operatorname{inc}(P)) \geqslant 0$ for all $(\mathbf{3}+\mathbf{1})$-free posets $P$,

7. $\theta\left(\widetilde{C}_{w}(1)\right) \geqslant 0$ for all 312-avoiding permutations $w \in \mathfrak{S}_{n}$,

8. $\theta\left(\widetilde{C}_{w}(1)\right) \geqslant 0$ for all 3412-avoiding, 4231-avoiding permutations $w \in \mathfrak{S}_{n}$

satisfy the implications (1) $\Rightarrow(2) \Rightarrow(4) \Rightarrow(5) \Leftrightarrow(6) \Leftrightarrow(7) \Leftrightarrow$ (8), and (3) $\Rightarrow$ (4).

Proof. $\quad((1) \Rightarrow(2) \Rightarrow(4))$ Suppose that (4) is false, i.e., that for some totally nonnegative matrix $A$ we have $\operatorname{Imm}_{\theta}(A)<0$. By Theorem 18, $A$ is the path matrix of a planar network $D$. Then Proposition 19 implies that some bijective skeleton $K$ of $D$ satisfies $\theta(z(K))<0$. It is straightforward to show that $z(K)$ equals a (positive rational multiple of a) product of Kazhdan-Lusztig basis elements of the form $\widetilde{C}_{w^{(1)}}(1) \cdots \widetilde{C}_{w^{(k)}}(1)$ in which each permutation $w^{(i)}$ is a maximal element of a parabolic subgroup of $\mathfrak{S}_{n}$. (See, e.g., $[9$, Cor. 5.3].) Thus (2) is false. This product in turn equals a nonnegative linear combination of Kazhdan-Lusztig basis elements. (See [16, Appendix].) Thus $\theta\left(\widetilde{C}_{w}(1)\right)<0$ for some $w \in \mathfrak{S}_{n}$ and (1) is false.

$((4) \Rightarrow(5))$ For every unit interval order $P$, there exists a planar network $D(P)$ with path matrix $A$ satisfying $\theta(\operatorname{inc}(P))=\operatorname{Imm}_{\theta}(A)$ [8, Prop. 3.8, Thm. 4.1, Cor. 7.5].

$((5) \Leftrightarrow(6))$ Each unit interval order is $(\mathbf{3}+\mathbf{1})$-free. Conversely, for each $(\mathbf{3}+\mathbf{1})$-free poset $P$, we have by $\left[15\right.$, Thm. 5.3] that $X_{\operatorname{inc}(P)}$ belongs to the cone generated by chromatic symmetric functions of unit interval orders.

$((5) \Rightarrow(8))$ By $[28$, Thm. 3.5, Lem. 5.3] and [8, Thm. 7.4], we have that for each 3412avoiding, 4231-avoiding permutation $w \in \mathfrak{S}_{n}$ there exists a unit interval order $P=P(w)$ satisfying $\theta\left(\operatorname{inc}(P(w))=\theta\left(\widetilde{C}_{w}(1)\right)\right.$ for all $\theta \in \mathcal{T}_{n}$.

$((8) \Rightarrow(7) \Rightarrow(5))$ Each 312-avoiding permutation also avoids the patterns 3412 and 4231 . The bijection (17) to unit interval orders and Proposition 5 give the last implication.

$((3) \Rightarrow(4))$ Follows from (53). 
Another consequence of Theorem 20 is an analog of Lemma 6 (2) for matrices. (See also [36, Prop. 2.4].)

Corollary 24. For $\theta_{1} \in \mathcal{T}_{k}, \theta_{2} \in \mathcal{T}_{n-k}, \theta=\theta_{1} \otimes \theta_{2} \uparrow_{\mathfrak{S}_{k} \times \mathfrak{S}_{n-k}}^{\mathfrak{S}_{n}} \in \mathcal{T}_{n}$, we have

$$
\operatorname{Imm}_{\theta}(A)=\sum_{J \subseteq[n]} \operatorname{Imm}_{\theta_{1}}\left(A_{J, J}\right) \operatorname{Imm}_{\theta_{2}}\left(A_{\bar{J}, \bar{J}}\right) .
$$

Proof. Similar to proof of Lemma 6 (2).

\subsection{Irreducible character immanants}

While no combinatorial interpretation is known for $\operatorname{Imm}_{\chi^{\lambda}}(A)$, Stembridge [35, Cor. 3.3] proved the following.

Theorem 25. For $\lambda \vdash n$ and an $n \times n$ totally nonnegative matrix $A$, we have

$$
\operatorname{Imm}_{\chi^{\lambda}}(A) \geqslant 0
$$

Problem 26. Combinatorially interpret the numbers $\operatorname{Imm}_{\chi^{\lambda}}(A)$ in Theorem 25.

We do have a combinatorial interpretation in the special case that $\lambda$ is a hook shape.

Theorem 27. For $A$ the path matrix of planar network $D$ of order $n$ and $k \leqslant n$, we have

$$
\operatorname{Imm}_{\chi^{k 1^{n-k}}}(A)=\sum_{\pi \in \mathcal{P}_{e}(D)} \operatorname{wgt}(\pi)\left(\# \text { standard } P(\pi) \text {-tableaux of shape } k 1^{n-k}\right) .
$$

In particular, when $k=n$, we obtain (55).

Proof. Let $\lambda=k 1^{n-k}$. By Proposition 19, Corollary 21, and Proposition 8, we have

$$
\begin{aligned}
\operatorname{Imm}_{\chi^{\lambda}}(A) & =\sum_{K} \operatorname{wgt}(K) \chi^{\lambda}(z(K)) \\
& =\sum_{K} \operatorname{wgt}(K) \sum_{\pi \in \Pi_{e}(K)} \chi^{\lambda}(P(\pi)) \\
& =\sum_{K} \operatorname{wgt}(K) \sum_{\pi \in \Pi_{e}(K)} \# \text { standard } P(\pi) \text {-tableaux of shape } \lambda,
\end{aligned}
$$

where $K$ varies over all bijective skeletons in $D$. This is equal to the claimed expression.

The case $k=n$ (55) can also be deduced from Stanley's interpretation [30, Thm. 3.3] of $\chi^{n}(\operatorname{inc}(P))$ as the number of acyclic orientations of inc $(P)$, using the bijection at the end of the proof of Theorem 10 .

Returning to Corollary 23, we see that for all $\lambda \vdash n$, Haiman's result [16, Lem. 1.1] that $\chi_{q}^{\lambda}\left(\widetilde{C}_{w}(q)\right) \in \mathbb{N}[q]$ for all $w \in \mathfrak{S}_{n}$ implies Stembridge's result [36, Cor. 3.3] that 
$\operatorname{Imm}_{\chi^{\lambda}}(A) \geqslant 0$ for all $A$ totally nonnegative, which in turn implies Gasharov's result [14] that $\chi^{\lambda}(\operatorname{inc}(P)) \geqslant 0$ for all $(\mathbf{3}+\mathbf{1})$-free posets $P$. The failure of the inequality $\chi^{\lambda}(\operatorname{inc}(P)) \geqslant$ 0 to hold for all posets $P$ and the equation (53) suggest that one might solve Problem 26 by explaining why for each bijective skeleton $K$ in a planar network, we have

$$
\sum_{\pi \in \Pi_{e}(K)} \chi^{\lambda}\left(\operatorname{inc}(P(\pi)) \geqslant 0, \quad \text { equivalently, } \quad \sum_{\pi \in \Pi_{e}(K)} X_{\operatorname{inc}(P(\pi))}\right. \text { is Schur-positive, }
$$

even when some of the posets $\left\{P(\pi) \mid \pi \in \Pi_{e}(K)\right\}$ are not $(\mathbf{3}+\mathbf{1})$-free.

\subsection{The permanent and induced trivial characters}

An obvious consequence of Proposition 19 is a combinatorial interpretation of the permanent of a totally nonnegative matrix.

Observation 28. Let totally nonnegative matrix $A$ be the path matrix of planar network D. Then we have

$$
\operatorname{per}(A)=\sum_{\pi \in \mathcal{P}(D)} \operatorname{wgt}(\pi)
$$

where $\operatorname{wgt}(\pi)$ is defined as in (47).

Two more combinatorial interpretations make use of the partial orders $\{P(\pi) \mid \pi \in$ $\left.\mathcal{P}_{e}(D)\right\}$. We have shown in Theorem 27 and will show in Theorem 30 that

$$
\begin{aligned}
\operatorname{per}(A) & =\sum_{\pi \in \mathcal{P}_{e}(D)} \operatorname{wgt}(\pi)(\# P(\pi) \text {-descent-free permutations of } \pi) \\
& =\sum_{\pi \in \mathcal{P}_{e}(D)} \operatorname{wgt}(\pi)(\# P(\pi) \text {-excedance-free permutations of } \pi) .
\end{aligned}
$$

Unfortunately we do not know how to obtain bijective proofs of these facts directly from Observation 28.

Problem 29. Given a planar network $D$ of order $n$, state an explicit bijection between path families in $D$ of arbitrary type, and $P$-descent-free $P(\pi)$-permutations, where $\pi$ in $D$ has type $e$, or $P$-excedance-free $P(\pi)$-permutations, where $\pi$ in $D$ has type $e$,

$$
\begin{aligned}
& \mathcal{P}(D) \quad \stackrel{1-1}{\longleftrightarrow} \bigcup_{\pi \in \mathcal{P}_{e}(D)}\left\{U \text { a } P(\pi) \text {-permutation } \mid \operatorname{des}_{P(\pi)}(U)=0\right\}, \\
& \mathcal{P}(D) \quad \stackrel{1-1}{\longleftrightarrow} \bigcup_{\pi \in \mathcal{P}_{e}(D)}\left\{U \text { a } P(\pi) \text {-permutation } \mid \operatorname{exc}_{P(\pi)}(U)=0\right\} .
\end{aligned}
$$

On the other hand, Theorem 27 and Proposition 9 easily prove (56).

Theorem 30. Let $A$ be the path matrix of planar network $D$ of order $n$, Then we have

$$
\operatorname{per}(A)=\sum_{\pi \in \mathcal{P}_{e}(D)} \operatorname{wgt}(\pi)(\# P(\pi) \text {-excedance-free permutations of } \pi) \text {. }
$$


Proof. Theorem 27 implies (55), and Proposition 9 then implies (56).

Now we have three combinatorial interpretations of induced trivial character immanants. To state these, we define more generalizations of Young tableaux. Given a path family $\pi=\left(\pi_{1}, \ldots, \pi_{n}\right) \in \mathcal{P}(D)$ for some planar network $D$, define a $\pi$-tableau of shape $\lambda$ to be a filling of a Young diagram with $\pi_{1}, \ldots, \pi_{n}$. For each $\pi$-tableau $U$, define $L(U)$ and $R(U)$ to be the Young tableaux whose integer entries are the source indices and sink indices, respectively, of the corresponding paths in $U$. Call the $\pi$-tableau $U$ left row-strict if entries of $L(U)$ increase from left to right in each row, and call it row-closed if $R\left(U_{i}\right)$ is a rearrangement of $L\left(U_{i}\right)$ for each $i$. For example, consider the planar network $D$ and path family $\pi \in \mathcal{P}(D)$,
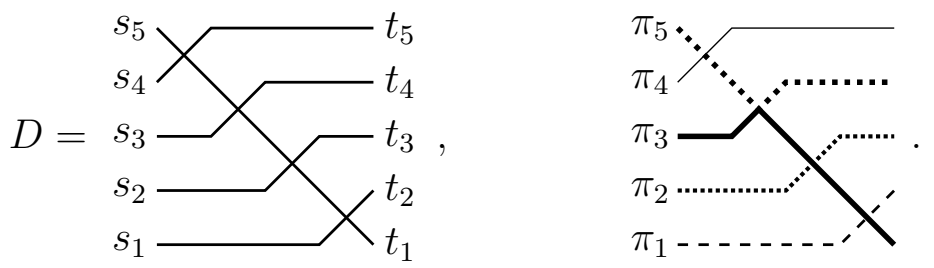

In order for a $\pi$-tableau to be row-closed and left row-strict, the paths $\pi_{1}, \pi_{2}, \pi_{3}$ must appear in order of increasing indices in the same row, as must $\pi_{4}, \pi_{5}$. All five paths must appear in order of increasing indices if they appear in a single row. There is one such $\pi$-tableau of shape 5 and one of shape 32. Together with their left and right Young tableaux, these are

$$
\begin{aligned}
& U=\begin{array}{l|l|l|l|l|l|}
\pi_{1} & \pi_{2} & \pi_{3} & \pi_{4} & \pi_{5}
\end{array}, \quad L(U)=\begin{array}{l|l|l|l|l|}
1 & 2 & 3 & 4 & 5
\end{array}, \quad R(U)=\begin{array}{ll|l|l|l|l|}
2 & 3 & 1 & 5 & 4
\end{array},
\end{aligned}
$$

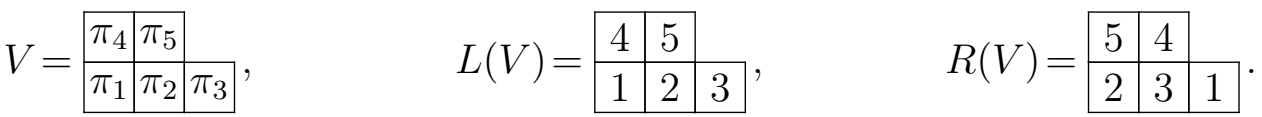

Theorem 31. Let $A$ be the path matrix of planar network $D$ of order $n$. Then for $\lambda \vdash n$, the evaluation $\operatorname{Imm}_{\eta^{\lambda}}(A)$ has the combinatorial interpretations

$$
\begin{aligned}
& \sum_{\pi \in \mathcal{P}(D)} \operatorname{wgt}(\pi)(\# \text { row-closed, left row-strict } \pi \text {-tableaux of shape } \lambda), \\
& \sum_{\pi \in \mathcal{P}_{e}(D)} \operatorname{wgt}(\pi)(\# \text { descent-free } P(\pi) \text {-tableaux of shape } \lambda), \\
& \sum_{\pi \in \mathcal{P}_{e}(D)} \operatorname{wgt}(\pi)(\# \text { excedance-free } P(\pi) \text {-tableaux of shape } \lambda) .
\end{aligned}
$$

Proof. Express $\operatorname{Imm}_{\eta^{\lambda}}(A)$ as in (50). By Observation 28, the term in this sum corresponding to a fixed ordered set partition $\left(I_{1}, \ldots, I_{r}\right)$ is equal to the sum of weights of path families $\pi=\left(\pi_{1}, \ldots, \pi_{n}\right)$ in which for $j=1, \ldots, r$, paths indexed by $I_{j}$ have sink indices also belonging to $I_{j}$. Each such partitioned path family $\pi$ naturally forms a left 
row-strict, row-closed $\pi$-tableau $U=U\left(\pi, I_{1}, \ldots, I_{r}\right)$ of shape $\lambda$, if we place paths indexed by $I_{j}$ into row $j$, with path indices increasing from left to right. We may therefore express $\operatorname{Imm}_{\eta^{\lambda}}(A)$ as

$$
\sum_{K} \operatorname{wgt}(K) \sum_{\pi \in \Pi(K)} \#\left\{\left(I_{1}, \ldots, I_{r}\right) \mid U\left(\pi, I_{1}, \ldots, I_{r}\right) \text { is row-closed, left row-strict of shape } \lambda\right\} \text {, }
$$

i.e., as (58). Alternatively, by Theorems 27 and 30, the term in (50) corresponding to a fixed ordered set partition $\left(I_{1}, \ldots, I_{r}\right)$ is equal to

$$
\begin{aligned}
& \sum_{K} \operatorname{wgt}(K) \sum_{\pi \in \Pi_{e}(K)} \#\left\{\left(U_{1}, \ldots, U_{r}\right) \mid U_{j} \text { a descent-free permutation of }\left(\pi_{i}\right)_{i \in I_{j}}\right\} \\
= & \sum_{K} \operatorname{wgt}(K) \sum_{\pi \in \Pi_{e}(K)} \#\left\{\left(U_{1}, \ldots, U_{r}\right) \mid U_{j} \text { an excedance-free permutation of }\left(\pi_{i}\right)_{i \in I_{j}}\right\} .
\end{aligned}
$$

Thus $\operatorname{Imm}_{\eta^{\lambda}}(A)$ is also equal to (59) and (60).

\subsection{Power sum immanants}

Like the induced trivial character immanants $\left\{\operatorname{Imm}_{\eta^{\lambda}}(A) \mid \lambda \vdash n\right\}$, the power sum immanants $\left\{\operatorname{Imm}_{\psi^{\lambda}}(A) \mid \lambda \vdash n\right\}$ have some combinatorial interpretations which are closely related to chromatic symmetric function coefficients, and others which are related to path families in a planar network. Call a $\pi$-tableau $U$ of shape $\lambda$ cylindrical if in each row $U_{i}=U_{i, 1} \cdots U_{i, \lambda_{i}}$, we have $R\left(U_{i, 1} \cdots U_{i, \lambda_{i}}\right)=L\left(U_{i, 2} \cdots U_{i, \lambda_{i}} U_{i, 1}\right)$, i.e., each path begins where the preceding path in its row terminates. For example, let $\pi$ be the path family in (57). There are six cylindrical $\pi$-tableaux, all of shape 32 :

$$
\begin{array}{|l|l|l|l|l|l|l|l|l|l|l|l|l|l|l|}
\hline \pi_{4} & \pi_{5} & \pi_{5} & \pi_{4} & \pi_{4} & \pi_{5} \\
\hline \pi_{1} & \pi_{2} & \pi_{3}
\end{array}, \quad \begin{array}{|l|l|l|l|l|}
\pi_{5} & \pi_{4} & \\
\hline \pi_{1} & \pi_{2} & \pi_{3} \\
\hline \pi_{2} & \pi_{3} & \pi_{1} \\
\hline \pi_{2} & \pi_{3} & \pi_{1} \\
\hline \pi_{3} & \pi_{1} & \pi_{2} \\
\hline
\end{array}, \quad \begin{array}{|l|l|l|}
\pi_{5} & \pi_{4} \\
\hline \pi_{3} & \pi_{1} & \pi_{2} \\
\hline
\end{array} .
$$

Theorem 32. Let $A$ be the path matrix of planar network $D$ of order $n$. Then for $\lambda \vdash n$, the evaluation $\operatorname{Imm}_{\psi^{\lambda}}(A)$ has the combinatorial interpretations

$$
\begin{aligned}
& \sum_{\pi \in \mathcal{P}_{e}(D)} \operatorname{wgt}(\pi)(\# \text { cyclically row-semistrict } P(\pi) \text {-tableaux of shape } \lambda), \\
& \sum_{\pi \in \mathcal{P}_{e}(D)} \operatorname{wgt}(\pi)(\# P(\pi) \text {-record-free row-semistrict } P(\pi) \text {-tableaux of shape } \lambda), \\
& \sum_{\pi \in \mathcal{P}(D)} \operatorname{wgt}(\pi)(\# \text { cylindrical } \pi \text {-tableaux of shape } \lambda) .
\end{aligned}
$$

Proof. By Proposition 19 and Corollary 21, we have

$$
\operatorname{Imm}_{\psi^{\lambda}}(A)=\sum_{K} \operatorname{wgt}(K) \psi^{\lambda}(z(K))=\sum_{K} \operatorname{wgt}(K) \sum_{\pi \in \Pi_{e}(K)} \psi^{\lambda}(\operatorname{inc}(P(\pi))) .
$$


Thus by Proposition 13 we have the interpretations (61) and (62). By (6), we have

$$
\operatorname{Imm}_{\psi^{\lambda}}(A)=z_{\lambda} \sum_{\begin{array}{c}
w \in \mathfrak{S}_{n} \\
\operatorname{ctype}(w)=\lambda
\end{array}} a_{1, w_{1}} \cdots a_{n, w_{n}} .
$$

The interpretation (63) now follows from the definition of path matrix.

\subsection{Monomial immanants}

By Corollary 23, the fact that we do not know $\phi^{\lambda}(\operatorname{inc}(P))$ to be nonnegative for unit interval orders $P$ ([33, Conj. 5.5]) implies that we do not know monomial immanants to evaluate nonnegatively on totally nonnegative matrices. We have the following conjecture of Stembridge [36, Conj. 2.1].

Conjecture 33. For $\lambda \vdash n$ and $A$ totally nonnegative we have $\operatorname{Imm}_{\phi^{\lambda}}(A) \geqslant 0$.

This is the strongest possible conjecture for inequalities of the form $\operatorname{Imm}_{\theta}(A) \geqslant 0$ with $\theta \in \mathcal{T}_{n}$, since $\operatorname{Imm}_{\theta}(A) \geqslant 0$ for all totally nonnegative $A \in \operatorname{Mat}_{n \times n}(\mathbb{R})$ only if $\theta$ is a nonnegative linear combination of $\left\{\phi^{\lambda} \mid \lambda \vdash n\right\}$ [36, Prop. 2.3]. Stembridge proved two special cases of Conjecture 33 [36, Thms. $2.7-2.8]$.

Proposition 34. Let $A$ be the path matrix of a planar network $D$ of order $n$.

1. If $\lambda=21^{n-2}$ then $\operatorname{Imm}_{\phi^{\lambda}}(A) \geqslant 0$.

2. If $\lambda$ is the rectangular shape $r^{k}$ then

$$
\operatorname{Imm}_{\phi^{\lambda}}(A)=\sum_{K} \operatorname{wgt}(\pi) \sum_{\pi \in \Pi(K)} \# \text { column-strict, cylindrical } \pi \text {-tableaux of shape } r^{k} .
$$

Since $\phi^{1^{n}}=\epsilon^{n}$ and $\phi^{n}=\psi^{n}$, one might hope that the formula (64) for $\operatorname{Imm}_{\phi^{\lambda}}(A)$, which combines aspects of Theorem 17 and Theorem 32, might hold for arbitrary $\lambda \vdash n$. Unfortunately, it does not. The planar network $D$ in (57) has path matrix

$$
A=\left[\begin{array}{lllll}
1 & 1 & 0 & 0 & 0 \\
1 & 1 & 1 & 0 & 0 \\
1 & 1 & 1 & 1 & 0 \\
1 & 1 & 1 & 1 & 1 \\
1 & 1 & 1 & 1 & 1
\end{array}\right]
$$

which satisfies

$$
\begin{aligned}
& \operatorname{Imm}_{\phi^{5}}(A)=5, \quad \operatorname{Imm}_{\phi^{41}}(A)=3, \quad \operatorname{Imm}_{\phi^{32}}(A)=7, \quad \operatorname{Imm}_{\phi^{221}}(A)=1, \\
& \operatorname{Imm}_{\phi^{311}}(A)=\operatorname{Imm}_{\phi^{2111}}(A)=\operatorname{Imm}_{\phi^{11111}}(A)=0 \text {. }
\end{aligned}
$$

Each of these monomial immanant evaluations, except for $\operatorname{Imm}_{\phi^{32}}(A)$, is consistent with the combinatorial interpretation given in (64). On the other hand, the path family $\pi$ 
in (57) is the unique path family covering $D$ which can be placed into a column-strict cylindrical path tableau of shape 32 . There are only four such tableaux:

$$
\begin{array}{|l|l|l|l|l|l|l|l|l|l|}
\hline \pi_{4} & \pi_{5} & \pi_{5} & \pi_{4} & \pi_{5} & \pi_{4} & \\
\hline \pi_{1} & \pi_{2} & \pi_{3} \\
\hline \pi_{1} & \pi_{2} & \pi_{3}
\end{array}, \quad \begin{array}{|l|l|l|l|}
\hline \pi_{4} & \pi_{5} & \\
\hline \pi_{2} & \pi_{3} & \pi_{1} \\
\hline \pi_{3} & \pi_{1} & \pi_{2} \\
\hline
\end{array} .
$$

The author has found that for $n \leqslant 5$, the sets of analogous tableaux for certain planar networks have cardinalities no greater than the true values of $\operatorname{Imm}_{\phi^{\lambda}}(A)$, where $A$ is the path matrix of the planar network. Specifically, these planar networks, called descending star networks in $[8, \S 3]$, correspond bijectively to unit interval orders. This suggests the following question.

Question 35. Let $P$ be a unit interval order on $n$ elements, and let $D=D(P)$ be the corresponding descending star network as defined in $[8, \S 3]$. Do we have for all $\lambda \vdash n$ that

$$
\phi^{\lambda}(\operatorname{inc}(P)) \geqslant \# \bigcup_{\pi \in \mathcal{P}(D)}\{U \mid U \text { a column-strict, cylindrical } \pi \text {-tableau of shape } \lambda\} ?
$$

As stated in Corollary 23, Stembridge's Conjecture 33 is a special case of Haiman's conjecture [16, Conj. 2.1] that $\phi_{q}^{\lambda}\left(\widetilde{C}_{w}(q)\right) \in \mathbb{N}[q]$ for all $w$. Furthermore, Haiman's conjecture would imply the equivalence of seven of the eight statements of Corollary 23.

Observation 36. If $\phi_{q}^{\lambda}\left(\widetilde{C}_{w}(q)\right) \in \mathbb{N}[q]$ for all $w$, then statements $(1),(2),(4), \ldots,(8)$ of Corollary 23 are all equivalent to the containment $\theta \in \operatorname{span}_{\mathbb{N}}\left\{\phi^{\lambda} \mid \lambda \vdash n\right\}$.

Proof. Assume that $\phi_{q}^{\lambda}\left(\widetilde{C}_{w}(q)\right) \in \mathbb{N}[q]$ for all $w$. Consider $\theta \in \mathcal{T}_{n}$ and expand it in the monomial trace basis as $\theta=\sum_{\lambda} a_{\lambda} \phi^{\lambda}$. If $a_{\lambda} \geqslant 0$ for all $\lambda$, then by our assumption we have

$$
\theta\left(\widetilde{C}_{w}(1)\right)=\sum_{\lambda} a_{\lambda} \phi^{\lambda}\left(\widetilde{C}_{w}(1)\right) \geqslant 0
$$

and statement (1) of Corollary 23 is true. Now suppose that $a_{\mu}<0$ for some $\mu \vdash n$ and let $w_{\mu}$ be the maximal element of the Young subgroup $\mathfrak{S}_{\mu}$. Then by (10) [16, Prop. 4.1] and our assumption we have that $\theta\left(\widetilde{C}_{w_{\mu}}(1)\right)<0$. Since $w_{\mu}$ avoids the patterns 3412 and 4231 , statement (8) of the Corollary is false.

\subsection{Immanant identities}

Analogous to Corollary 16 are three identities which follow from Corollary 24. The third of these is known as Muir's identity. 
Corollary 37. Let $A$ be an $n \times n$ matrix. We have

$$
\begin{gathered}
n \operatorname{per}(A)=\sum_{i=1}^{n} \sum_{\substack{J \\
|J|=i}} \operatorname{Imm}_{\psi^{i}}\left(A_{J, J}\right) \operatorname{per}\left(A_{\bar{J}, \bar{J}}\right), \\
n \operatorname{det}(A)=\sum_{i=1}^{n}(-1)^{i-1} \sum_{\substack{J \\
|J|=i}} \operatorname{Imm}_{\psi^{i}}\left(A_{J, J}\right) \operatorname{det}\left(A_{\bar{J}, \bar{J}}\right), \\
\sum_{i=0}^{n}(-1)^{i} \sum_{\substack{J \\
|J|=i}} \operatorname{det}\left(A_{J, J}\right) \operatorname{per}\left(A_{\bar{J}, \bar{J}}\right)=0,
\end{gathered}
$$

where the sums are over ordered set partitions of type $(i, n-i)$.

Proof. Similar to proof of Corollary 16.

\section{Applications to chromatic quasisymmetric functions}

Shareshian and Wachs [26] defined a quasisymmetric extension $X_{G, q}$ of Stanley's chromatic symmetric function $X_{G}(11)$. Let $G=(V, E)$ be a simple directed graph on vertices labeled $1, \ldots, n$. Given a proper coloring $\kappa: V \rightarrow\{1,2, \ldots$,$\} of G$, define $\operatorname{inv}_{G}(\kappa)$ to be the number of pairs $(i, j) \in E$ with $i<j$ and $\kappa(i)>\kappa(j)$. For any composition $\alpha=\left(\alpha_{1}, \ldots, \alpha_{\ell}\right) \vDash n$, define

$$
c(G, \alpha, q)=\sum_{\substack{\kappa \\ \operatorname{type}(\kappa)=\alpha}} q^{\operatorname{inv}_{G}(\kappa)},
$$

and let

$$
M_{\alpha}=\sum_{i_{1}<\cdots<i_{\ell}} x_{i_{1}}^{\alpha_{1}} \cdots x_{i_{\ell}}^{\alpha_{\ell}}
$$

be the monomial quasisymmetric function indexed by $\alpha$. Then we have the definition

$$
X_{G, q}=\sum_{\kappa} q^{\operatorname{inv}_{G}(\kappa)} x_{\kappa(1)} \cdots x_{\kappa(n)}=\sum_{\alpha \models n} c(G, \alpha, q) M_{\alpha}
$$

where the first sum is over all proper colorings of $G$. It is easy to see that we have $X_{G, 1}=X_{G}$.

In the special case that $X_{G, q}$ is symmetric, Proposition 3 implies that there is an element $g \in \mathbb{Q}(q) \otimes H_{n}(q)$ satisyfing $\epsilon_{q}^{\lambda}(g)=c(G, \alpha, q)$ for every rearrangement $\alpha$ of $\lambda$. Thus for all $\theta_{q} \in \mathcal{T}_{n, q}$ we may define

$$
\theta_{q}(G):=\theta_{q}(g)
$$


to obtain a $q$-extension

$$
\begin{gathered}
X_{G, q}=\sum_{\lambda \vdash n} \epsilon_{q}^{\lambda}(G) m_{\lambda}=\sum_{\lambda \vdash n} \eta_{q}^{\lambda}(G) f_{\lambda}=\sum_{\lambda \vdash n} \frac{(-1)^{n-\ell(\lambda)} \psi_{q}^{\lambda}(G)}{z_{\lambda}} p_{\lambda}=\sum_{\lambda \vdash n} \chi_{q}^{\lambda^{\top}}(G) s_{\lambda} \\
=\sum_{\lambda \vdash n} \phi_{q}^{\lambda}(G) e_{\lambda}=\sum_{\lambda \vdash n} \gamma_{q}^{\lambda}(G) h_{\lambda}
\end{gathered}
$$

of (13) and a similar $q$-extension of (14) for $\omega X_{G, q}$.

Shareshian and Wachs showed that when $P$ is a unit interval order labeled as in (16), the function $X_{\operatorname{inc}(P), q}$ is in fact symmetric. By [8, Cor. 7.5], the coefficients

$$
\epsilon_{q}^{\lambda}(\operatorname{inc}(P)), \quad \eta_{q}^{\lambda}(\operatorname{inc}(P)), \quad \chi_{q}^{\lambda}(\operatorname{inc}(P)), \quad \psi_{q}^{\lambda}(\operatorname{inc}(P)), \quad \phi_{q}^{\lambda}(\operatorname{inc}(P)), \quad \gamma_{q}^{\lambda}(\operatorname{inc}(P))
$$

in each standard expansion of $X_{\operatorname{inc}(P), q}$ are given by $\theta_{q}(\operatorname{inc}(P))=\theta_{q}\left(\widetilde{C}_{w}(q)\right)$ for the 312avoiding permutation $w$ related to $P$ as in (17). For interpretations of some of these coefficients, see $[8, \S 5-10],[27, \S 5-7]$. Shareshian and Wachs conjectured [26, Conj. 5.1] nonnegativity of the elementary coefficients of $X_{\mathrm{inc}(P), q}$. This extends the Stanley-Stembridge conjecture [33, Conj. 5.5] that $\phi^{\lambda}(\operatorname{inc}(P)) \geqslant 0$, and is equivalent to a special case of Haiman's conjecture $\left[16\right.$, Conj. 2.1] that $\phi_{q}^{\lambda}\left(\widetilde{C}_{w}(q)\right) \in \mathbb{N}[q]$ for all $w$. (For progress on this conjecture, see [10], [17] and references there.)

Conjecture 38. For $P$ a unit interval order labeled as in $(16)$, we have $\phi_{q}^{\lambda}(\operatorname{inc}(P)) \in \mathbb{N}[q]$.

Since $\sum_{\lambda \vdash n} \phi_{q}^{\lambda}=\eta_{q}^{n}$, the sum $\sum_{\lambda \vdash n} \phi_{q}^{\lambda}(\operatorname{inc}(P))=\eta_{q}^{n}(\operatorname{inc}(P))$ satisfies two identities which extend (34) when $P$ is a unit interval order labeled as in (16).

1. Given an acyclic orientation $O$, define $\operatorname{inv}(O)$ to be the number of oriented edges $(j, i)$ with $j>i$. We have

$$
\sum_{\lambda \vdash n} \phi_{q}^{\lambda}(\operatorname{inc}(P))=\sum_{O} q^{\operatorname{inv}(O)}
$$

where the sum on the right is over all acyclic orientations $O$ of inc $(P)$ [26, Thm. 5.3].

2. Given a $P$-permutation $U$, define $\operatorname{inv}_{P}(U)=\#\left\{(i, j) \mid j>i\right.$ (as integers), $j \ngtr_{P} i$, and $j$ appears to the left of $i$ in $\left.U\right\}$. We have

$$
\sum_{\lambda \vdash n} \phi_{q}^{\lambda}(\operatorname{inc}(P))=\sum_{U} q^{\operatorname{inv}_{P}(U)},
$$

where the sum is over all $P$-descent-free $P$-permutations $U$ [26, Thm. 6.3].

It is possible to extend Theorem $10(2)$ to obtain a similar formula for excedance-free $P$-permutations as well. Given a $P$-tableau $U$, define

$$
\operatorname{inv}(U)=\#\{(i, j) \mid j>i \text { (as integers), and } j \text { appears to the left of } i \text { in } U\} \text {. }
$$


Proposition 39. Let $P$ be an n-element unit interval order, labeled as in (16) and corresponding by (17) to the 312-avoiding permutation $w \in \mathfrak{S}_{n}$. Then we have

$$
\sum_{\lambda \vdash n} \phi_{q}^{\lambda}(\operatorname{inc}(P))=\sum_{v \leqslant w} q^{\ell(v)}=\sum_{U} q^{\operatorname{inv}(U)},
$$

where $U$ in the final sum varies over all P-excedance-free P-permutations.

Proof. By [8, Cor. 7.5] and (4) we have

$$
\sum_{\lambda \vdash n} \phi_{q}^{\lambda}(\operatorname{inc}(P))=\eta_{q}^{n}(\operatorname{inc}(P))=\eta_{q}^{n}\left(\widetilde{C}_{w}(q)\right)=\eta_{q}^{n}\left(\sum_{v \leqslant w} T_{v}\right) .
$$

Since $\eta_{q}^{n}\left(T_{v}\right)=q^{\ell(v)}$, we have the first equality in (70). To see the second equality, let $U=v_{1} \cdots v_{n}$ be a $P$-permutation. Then $U$ appears in the third sum of (70) if and only if $\operatorname{exc}_{P}(U)=0$. By Corollary 11, this condition is equivalent to $v \leqslant w$, and clearly we have $\ell(v)=\operatorname{inv}(U)$.

Combining Proposition 39 with (68) and (69), we obtain equidistribution results for the three variations of inversion statistics. Unfortunately, the map $w \mapsto \sigma\left(w^{-1}\right)$ from Subsection 3.3, which satisfies $\operatorname{exc}_{P}(w)=\operatorname{des}_{P}\left(\sigma\left(w^{-1}\right)\right)$ does not satisfy $\operatorname{inv}(w)=\operatorname{inv}_{P}\left(\sigma\left(w^{-1}\right)\right)$. (Neither does the map in [34, Rmk. 4.7] mentioned before Proposition 9.) Furthermore, the statistic pairs $\left(\operatorname{exc}_{P}\right.$, inv $)$ and $\left(\operatorname{des}_{P}, \operatorname{inv}_{P}\right)$ cannot be equidistributed on $\mathfrak{S}_{n}$, since inv and $\operatorname{inv}_{P}$ are not equidistributed on $\mathfrak{S}_{n}$. This suggests the following problem for unit interval orders $P$, labeled as in (16).

Problem 40. Find a bijection $\varphi$ from descent-free $P$-permutations to excedance-free $P$-permutations which satisfies $\operatorname{inv}_{P}(U)=\operatorname{inv}(\varphi(U))$.

Just as Proposition 14 refines (34) and (35), Shareshian and Wachs [26, Thm. 5.3] refined (68), (69) as follows.

Proposition 41. For all unit interval orders $P$ labeled as in (16), we have that

$$
\sum_{\substack{\lambda \vdash n \\ \ell(\lambda)=k}} \phi_{q}^{\lambda}(\operatorname{inc}(P))=\sum_{U} q^{\operatorname{inv}_{P}(U)}=\sum_{O} q^{\operatorname{inv}(O)},
$$

where the second and third sum are over descent-free $P$-permutations $U$ having $k P$ records, and acyclic orientations $O$ of inc $(P)$ having $k$ sources.

It would be interesting to similarly refine Proposition 39 .

Problem 42. Let $P$ be an $n$-element unit interval order labeled as in $(16)$, and let $w(P)$ be the corresponding 312-avoiding permutation as in (17). Find functions $\delta_{1}, \delta_{2}$ so that

$$
\sum_{\substack{\lambda \vdash n \\ \ell(\lambda)=k}} \phi_{q}^{\lambda}(\operatorname{inc}(P))=\sum_{\substack{v \leqslant w \\ \delta_{1}(v, w)=k}} q^{\ell(v)}=\sum_{\substack{\operatorname{exc}_{P}(U)=0 \\ \delta_{2}(U)=k}} q^{\operatorname{inv}(U)},
$$

where $U$ in the final sum is a $P$-permutation. 
Finally, it would be interesting to combinatorially settle the Shareshian-Wachs conjecture $\left[26\right.$, Conj. 5.1] that $\phi_{q}^{\lambda}(\operatorname{inc}(P)) \in \mathbb{N}[q]$ as follows.

Problem 43. For each $n$-element unit interval order $P$ labeled as in (16), and each partition $\lambda \vdash n$, define subsets $\mathcal{S}_{1}(\lambda), \mathcal{S}_{2}(\lambda), \mathcal{S}_{3}(\lambda), \mathcal{S}_{4}(\lambda)$ of acyclic orientations of inc $(P), P$ descent-free $P$-permutations, $\left\{v \in \mathfrak{S}_{n} \mid v \leqslant w(P)\right\}$, and $P$-excedance free $P$-permutations, respectively, so that

$$
\phi_{q}^{\lambda}(\operatorname{inc}(P))=\sum_{O \in \mathcal{S}_{1}(\lambda)} q^{\operatorname{inv}(O)}=\sum_{U \in \mathcal{S}_{2}(\lambda)} q^{\operatorname{inv}_{P}(U)}=\sum_{v \in \mathcal{S}_{3}(\lambda)} q^{\ell(v)}=\sum_{U \in \mathcal{S}_{4}(\lambda)} q^{\operatorname{inv}(U)} .
$$

\section{Acknowledgements}

The author is grateful to an anonymous referee for numerous corrections and suggestions which helped improve the article significantly.

\section{References}

[1] C. A. Athanasiadis. Power sum expansion of chromatic quasisymmetric functions. Electron. J. Combin., 22(2): \#P2.7, 2015.

[2] D. Beck, J. Remmel, and T. Whitehead. The combinatorics of transition matrices between the bases of the symmetric functions and the $B_{n}$ analogues. Discrete Math., 153:3-27, 1996.

[3] A. Björner and F. Brenti. Combinatorics of Coxeter groups, volume 231 of Graduate Texts in Mathmatics. Springer, New York, 2005.

[4] F. Brenti. Combinatorics and total positivity. J. Combin. Theory Ser. A, 71(2):175218, 1995.

[5] L. Carlitz, R. Scoville, and T. Vaughan. Enumeration of pairs of sequences by rises, falls, and levels. Manuscripta Math., 19:211-243, 1976.

[6] S. Cho and J. Hong. Positivity of chromatic symmetric functions associated with Hessenberg functions of bounce number 3. arXiv:1910.07308v1, 2019.

[7] T. Chow. Descents, quasi-symmetric functions, Robinson-Schensted for posets, and the chromatic symmetric function. J. Algebraic Combin., 10:227-240, 1999.

[8] S. Clearman, M. Hyatt, B. Shelton, and M. Skandera. Evaluations of Hecke algebra traces at Kazhdan-Lusztig basis elements. Electron. J. Combin., 23(2): \#P2.7, 2016.

[9] A. Clearwater and M. Skandera. Total nonnegativity and Hecke algebra trace evaluations. arXiv:2007.14512v1, 2019.

[10] L. Colmenarejo, A. Morales, and G. Panova. Chromatic symmetric functions of Dyck paths and q-rook theory. arXiv:2012.00913, 2020.

[11] C. W. Cryer. Some properties of totally positive matrices. Linear Algebra Appl., 15:1-25, 1976. 
[12] S. Dahlberg. A new formula for Stanley's chromatic symmetric function for unit interval graphs and e-positivity for triangular ladder graphs. Sém. Lothar. Combin., 82: Article B59, 12 pages, 2019.

[13] E. Egge, N. Loehr, and G. Warrington. From quasisymmetric expansions to Schur expansions via a modified inverse Kostka matrix. European J. Combin., 31:20142027, 2010.

[14] V. Gasharov. Incomparability graphs of $(\mathbf{3}+\mathbf{1})$-free posets are $s$-positive. Discrete Math., 157:211-215, 1996.

[15] M. Guay-Paquet. A modular relation for the chromatic symmetric functions of $(\mathbf{3}+\mathbf{1})$-free posets. arXiv:1306.2400, 2013.

[16] M. Haiman. Hecke algebra characters and immanant conjectures. J. Amer. Math. Soc., 6(3):569-595, 1993.

[17] M. Harada, M. Precup, and J. Tymoczko. Permutation bases in the equivariant cohomology rings of regular semisimple Hessenberg varieties. arXiv:2101.03191, 2021.

[18] R. Kaliszewski. Hook coefficients of chromatic functions. J. Comb., 6(3):327-337, 2015.

[19] S. Karlin and G. McGregor. Coincidence probabilities. Pacific J. Math., 9:1141-1164, 1959.

[20] D. Kazhdan and G. Lusztig. Representations of Coxeter groups and Hecke algebras. Invent. Math., 53:165-184, 1979.

[21] B. Lindström. On the vector representations of induced matroids. Bull. London Math. Soc., 5:85-90, 1973.

[22] D. E. Littlewood. The Theory of Group Characters and Matrix Representations of Groups. Oxford University Press, New York, 1940.

[23] C. Loewner. On totally positive matrices. Math. Z., 63:338-340, 1955.

[24] I. Macdonald. Symmetric Fuctions and Hall Polynomials. Oxford University Press, Oxford, 1979.

[25] R. Merris and W. Watkins. Inequalities and identities for generalized matrix functions. Linear Algebra Appl., 64:223-242, 1985.

[26] J. Shareshian and M. Wachs. Chromatic quasisymmetric functions and Hessenberg varieties. In Configuration Spaces, pages 433-460. Edizione Della Normale, Pisa, 2012.

[27] J. Shareshian and M. L. Wachs. Chromatic quasisymmetric functions. Adv. Math., 295:497-551, 2016.

[28] M. Skandera. On the dual canonical and Kazhdan-Lusztig bases and 3412, 4231avoiding permutations. J. Pure Appl. Algebra, 212, 2008.

[29] M. Skandera. Graph coloring and hyperoctahedral group character evaluations 2021. In preparation. 
[30] R. Stanley. A symmetric function generalization of the chromatic polynomial of a graph. Adv. Math., 111:166-194, 1995.

[31] R. Stanley. Enumerative Combinatorics, vol. 1. Cambridge University Press, Cambridge, 1997.

[32] R. Stanley. Enumerative Combinatorics, vol. 2. Cambridge University Press, Cambridge, 1999.

[33] R. Stanley and J. R. Stembridge. On immanants of Jacobi-Trudi matrices and permutations with restricted positions. J. Combin. Theory Ser. A, 62:261-279, 1993.

[34] E. Steingrímsson. Permutation Statistics of Indexed and Poset Permutations. Ph.D. thesis, MIT, Cambridge, MA 1992.

[35] J. Stembridge. Immanants of totally positive matrices are nonnegative. Bull. London Math. Soc., 23:422-428, 1991.

[36] J. Stembridge. Some conjectures for immanants. Canad. J. Math., 44(5):1079-1099, 1992.

[37] A. Whitney. A reduction theorem for totally positive matrices. J. Anal. Math., 2:88-92 1952. 